\title{
Gold-catalyzed Intermolecular Hetero-Dehydro-Diels-Alder Cycloaddition of Captodative Dienynes with Nitriles: A New Reaction and a Regioselective Direct Access to Pyridines
}

\author{
José Barluenga, ${ }^{*}$ Manuel Ángel Fernández-Rodríguez, Patricia García-García, and Enrique Aguilar* \\ Instituto Universitario de Química Organometálica “Enrique Moles”, Unidad Asociada al CSIC, Universidad de \\ Oviedo, Julián Clavería 8, 33006-Oviedo, Spain and Instituto de Investigaciones Químicas y Ambientales de \\ Barcelona (IIQAB)-CSIC, Jordi Girona 18-26, 08034-Barcelona, Spain
}

\section{Supporting Information}

General Considerations: All reactions involving air sensitive compounds were carried out under a $\mathrm{N}_{2}$ atmosphere (99.99\%). All glassware was oven-dried $\left(120{ }^{\circ} \mathrm{C}\right)$, evacuated and purged with nitrogen. All common reagents and solvents were obtained from commercial suppliers and used without any further purification unless otherwise indicated. Captodative 1,3-dien-5-ynes 1a,c,d,f,g and $\mathbf{7}$ have previously reported and were prepared following described procedures. ${ }^{1}$ Enynes $4^{2}$ and $5^{3}$ have been previously described. Commercial nitriles were distilled prior to use. Both components of the catalytic system $\left(\mathrm{AgSbF}_{6}\right.$ and $\mathrm{AuClPEt}_{3}$ ) were acquired from commercial suppliers. 1,2Dichloroethane, toluene, THF and 1,2-dimethoxyethane were dried by standard methods. TLC was performed on aluminumbacked plates coated with silica gel 60 with $\mathrm{F}_{254}$ indicator; the chromatograms were visualized under ultraviolet light and/or by staining with a $\mathrm{Ce} / \mathrm{Mo}$ reagent, potassium permanganate or phosphomolibdic acid solutions and subsequent heating. $\mathrm{R}_{f}$ values are reported on silica gel. Flash column chromatography was carried out on silica gel 60, 230-240 mesh. Routine NMR measurements were recorded on Bruker AC-300, DPX-300 or AV-400 spectrometers. ${ }^{1} \mathrm{H}$ NMR: splitting pattern abbreviations are: s, singlet; bs, broad singlet; d, doublet; t, triplet; q, quartet; hex, hextuplet; hept, heptuplet; $m$, multiplet. ${ }^{13} \mathrm{C}$ NMR: multiplicities were determined by DEPT, abbreviations are: q, $\mathrm{CH}_{3}$; t, $\mathrm{CH}_{2}$; d, $\mathrm{CH}$; s, quaternary carbons. COSY, HSQC, HMBC and NOESY experiments were carried out on Bruker DPX-300 or AV-400 spectrometer. Standard pulse sequences were employed for the DEPT experimentss. FT-IR were performed with a Mattson 3000 FT-IR spectrometer. Mass spectra were determined by Universidad de Oviedo and Universidad de Vigo (CACTI) services, with a Finnigan Mat95, and a VG AutoSpec M Mass Spectrometers respectively for high resolution mass spectra (HRMS); low resolution mass spectra were obtained with a Hewlett-Packard 5880 A Spectrometer. Electron impact $(70 \mathrm{eV})$ or fast atom bombardment (FAB) techniques were employed. Melting points were determined on a Büchi-Tottoli apparatus and are uncorrected.

General procedure for the synthesis of captodative 1,3dien-5-ynes 1b,e. ${ }^{1}$ 2-Methoxyfuran (2 equiv) was added to a solution of the corresponding carbene complex (1 equiv, $1 \mathrm{mmol}$ ) in dry THF $(10 \mathrm{~mL})$ at $0^{\circ} \mathrm{C}$. The temperature was raised to r.t. and

\footnotetext{
${ }^{1}$ Barluenga, J.; García-García, P.; de Sáa, D.; Fernández-Rodríguez, M.A.; Bernardo de la Rúa, R.; Ballesteros, A.; Aguilar, E.; Tomás, M. Angew. Chem. Int. Ed. 2007, 46, 2610-2612.

${ }_{3}^{2}$ Kang, S.-K.; Kim, W.-Y.; Jiao, X. Synthesis 1998, 1252-1254.

Takeuchi, R.; Tanabe, K.; Tanaka, S. J. Org. Chem. 2000, 65, 1558-1561.
}

the mixture stirred until complete disappearance of the carbene complex was observed by TLC (6-12 h). Solvent was removed under reduced pressure, and the residue purified by flash chromatography (hexane/AcOEt); the corresponding dienyne $\mathbf{1 b}$ or 1e was isolated in the yield reported below.

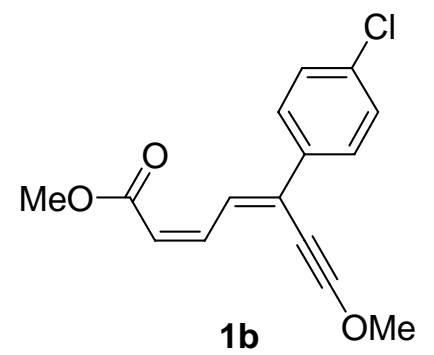

Dienyne 1b. 72\% Yield. Yellow oil. $\mathrm{R}_{f}=0.37$ (Hexane/AcOEt : 5/1). IR $v\left(\mathrm{~cm}^{-1}\right)$ 2249, 1712, 1602. ${ }^{1} \mathrm{H}$ NMR $\left(\mathrm{CDCl}_{3}, 400 \mathrm{MHz}\right)$ $\delta 8.21$ (d, $J=11.6 \mathrm{~Hz}, 1 \mathrm{H}), 7.69$ (d, $J=8.4 \mathrm{~Hz}, 2 \mathrm{H}), 7.34-7.25$ (m, $3 \mathrm{H}), 5.81$ (d, $J=11.6 \mathrm{~Hz}, 1 \mathrm{H}), 4.08$ (s, $3 \mathrm{H}), 3.76$ (s, $3 \mathrm{H})$. ${ }^{13} \mathrm{C} \mathrm{NMR}\left(\mathrm{CDCl}_{3}, 75 \mathrm{MHz}\right) \delta 167.1$ (s), 142.2 (d), 136.7 (s), 134.7 (s), 130.8 (s), 128.5 (d, 2 CH), 128.1 (d, 2 CH), 127.2 (d), 117.8 (d), 110.7 (s), 66.6 (q), 51.2 (q), 36.3 (s).

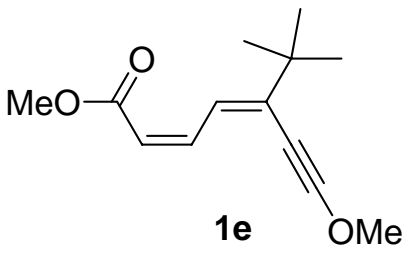

Dienyne 1e. $86 \%$ Yield. Yellow oil. $\mathrm{R}_{f}=0.45$ (Hexane/AcOEt : 5/1). IR $v\left(\mathrm{~cm}^{-1}\right) 2238,1713,1610 .{ }^{1} \mathrm{H} \mathrm{NMR}\left(\mathrm{CDCl}_{3}, 400 \mathrm{MHz}\right)$ $\delta 7.54$ (d, $J=11.4 \mathrm{~Hz}, 1 \mathrm{H}), 7.10$ (t, $J=11.4 \mathrm{~Hz}, 1 \mathrm{H}), 5.61$ (d, $J$ = $11.4 \mathrm{~Hz}, 1 \mathrm{H}), 3.96$ (s, $3 \mathrm{H}), 3.67$ (s, $3 \mathrm{H}), 1.14$ (s, $9 \mathrm{H}) .{ }^{13} \mathrm{C}$ NMR (CDCl, $75 \mathrm{MHz}) \delta 167.1$ (s), 144.9 (s), 143.5 (d), 124.1 (d), 115.8 (d), 110.7 (s), 66.3 (q), 50.9 (q), 37.0 (s), 36.5 (s), 29.2 (q, $3 \mathrm{CH}_{3}$ ). MS (m/z) $222\left(\mathrm{M}^{+}, 14\right), 207$ (15), 191 (14), 163 (60), 151 (97), 57 (100). HRMS (EI) calcd for $\mathrm{C}_{13} \mathrm{H}_{18} \mathrm{O}_{3}[\mathrm{M}]^{+}$, 222.1251. Found: 222.1247.

Formation of dimer 2a. 1,3-Dien-5-yne 1a evolves spontaneously when neat to yield dimer $\mathbf{2 a}$. The reaction does not go to completion, even at long reaction times, but the dimer is easily separated from the starting dienyne by flash column chromatography on silica gel (hexane/AcOEt : 5/1 as eluent). 
The formation of $\mathbf{2 a}$ was also observed when 1a was heated at $85{ }^{\circ} \mathrm{C}$ or treated with different catalytic systems (Pt, Cu, Ag, Pd) in the presence of acetonitrile.

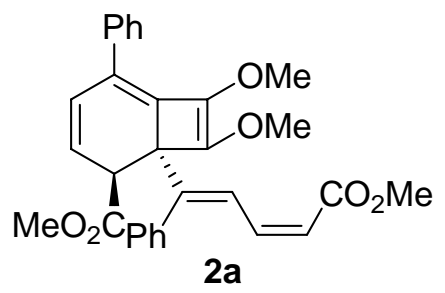

\section{Methyl} (methoxycarbonyl)-1-phenylbuta-1,3-dienyl]-5-

phenylbicyclo[4.2.0]octa-3,5,7-triene-2-carboxylate. Dimer 2a: $33 \%$ Yield. Yellow oil. $\mathrm{R}_{f}=0.14$ (Hexane/AcOEt : 5/1). IR $v$ $\left(\mathrm{cm}^{-1}\right)$ 1737, 1711, 1615. ${ }^{1} \mathrm{H}$ NMR $\left(\mathrm{CDCl}_{3}, 300 \mathrm{MHz}\right) \delta$ 7.46-7.39 (m, $2 \mathrm{H}), 7.32-7.13(\mathrm{~m}, 10 \mathrm{H}), 6.32$ (d, $J=8.9 \mathrm{~Hz}, 1 \mathrm{H})$, 5.875.78 (m, 2 H), 4.14 (s, $3 \mathrm{H}), 3.79$ (d, $J=7.0 \mathrm{~Hz}, 1 \mathrm{H}), 3.71$ (s, 3 $\mathrm{H}), 3.64$ (s, $3 \mathrm{H}), 3.25$ (s, $3 \mathrm{H}) .{ }^{13} \mathrm{C} \mathrm{NMR}\left(\mathrm{CDCl}_{3}, 75 \mathrm{MHz}\right) \delta$ 170.7 (s), 166.7 (s), 150.6 (s), 149.6 (s), 141.8 (s), 140.3 (d), 138.0 (s), 132.6 (s), 131.5 (s), 131.0 (d), 128.0 (d, 2 CH), 127.9 (d), 127.5 (d, 2 CH), 127.0 (d), 126.8 (d, 2 CH), 126.6 (d, 2 CH), 126.3 (d), 121.8 (d), 117.7 (s), 117.4 (d), 61.2 (q), 59.3 (q), 51.7 (q), 51.1 (q), 49.31 (s), 49.28 (d). MS (m/z) $484\left(\mathrm{M}^{+}, 100\right), 425$ (25), 393 (49), 289 (68). HRMS (EI) calcd for $\mathrm{C}_{30} \mathrm{H}_{28} \mathrm{O}_{6}[\mathrm{M}]^{+}$, 484.1880. Found: 484.1879.

Catalyst screening for the synthesis of pyridine 3a. The metal catalyst (5 mol\%) was added to a solution of dienyne 1a (0.2 mmol, $48 \mathrm{mg}$ ) in dry acetonitrile $(2 \mathrm{~mL})$. The reaction mixture was stirred at $85{ }^{\circ} \mathrm{C}$ until complete disappearance of dyenine was observed by TLC or GC/MS. Solvent was removed under reduced pressure and the yield of pyridine 3a was estimated by ${ }^{1} \mathrm{H}$ NMR (400 MHz) of the crude mixture adding ${ }^{t} \mathrm{BuOAc}(0.2$ mmol, $27 \mu \mathrm{L}$ ) as internal standard.

General procedure for the synthesis of pyridines 3 or 8 . $\mathrm{AuClPEt}_{3}$ (5 mol\%, $9 \mathrm{mg}$ ) and $\mathrm{AgSbF}_{6}$ (5 mol\%, $9 \mathrm{mg}$ ) were added to a solution of the corresponding dienyne $\mathbf{1}(0.5 \mathrm{mmol})$ and the appropriate nitrile (20 equiv., $10 \mathrm{mmol}$ ) in dry 1,2dichloroethane $(5 \mathrm{~mL})$. The reaction mixture was stirred at $85{ }^{\circ} \mathrm{C}$ until complete disappearance of dyenine was observed by TLC or GC/MS. Solvent was removed under reduced pressure and the crude mixture was purified by flash chromatography on silica gel using mixtures of hexane and AcOEt. The corresponding pyridines $\mathbf{3}$ or $\mathbf{8}$ were isolated in the yields reported in Table 2 and equation 1.<smiles>COC(=O)/C=C\c1c(-c2ccccc2)cc(OC)nc1C</smiles>

Pyridine 3a: $70 \%$ Yield. White solid. $\mathrm{Mp}=76-78{ }^{\circ} \mathrm{C} . \mathrm{R}_{f}=$ 0.33 (Hexane/AcOEt : 5/1). IR $v\left(\mathrm{~cm}^{-1}\right)$ 1727, 1590. ${ }^{1} \mathrm{H}$ NMR $\left(\mathrm{CDCl}_{3}, 400 \mathrm{MHz}\right) \delta$ 7.38-7.34 (m, $\left.3 \mathrm{H}\right), 7.30-7.27$ (m, $\left.2 \mathrm{H}\right), 6.90$ (d, $J=11.8 \mathrm{~Hz}, 1 \mathrm{H}), 6.56$ (s, $1 \mathrm{H}), 5.96$ (d, $J=11.8 \mathrm{~Hz}, 1 \mathrm{H})$, 3.97 (s, $3 \mathrm{H}$ ), 3.56 (s, $3 \mathrm{H}), 2.43$ (s, $3 \mathrm{H}) .{ }^{13} \mathrm{C} \mathrm{NMR}\left(\mathrm{CDCl}_{3}, 100\right.$ MHz) $\delta 165.7$ (s), 162.6 (s), 153.3 (s), 151.4 (s), 141.9 (d), 139.1 (s), 128.9 (d, 2 CH), 127.9 (d, 2 CH), 127.8 (d), 123.0 (d), 122.4 (s), 107.6 (d), 53.3 (q), 51.2 (q), 22.9 (q). MS (m/z) $283\left(\mathrm{M}^{+}, 27\right)$, 282 ([M-1] $\left.{ }^{+}, 54\right), 224$ (100), 222 (73), 194 (30). HRMS (EI) calcd for $\mathrm{C}_{17} \mathrm{H}_{17} \mathrm{NO}_{3}[\mathrm{M}]^{+}$, 283.1208. Found: 283.1201.<smiles>COC(=O)/C=C\c1c(-c2ccccc2)cc(OC)nc1Br</smiles>

Pyridine 3b: $64 \%$ Yield. Colorless oil. $\mathrm{R}_{f}=0.40$ (Hexane/AcOEt : 5/1). IR $v\left(\mathrm{~cm}^{-1}\right)$ 1727, 1588. ${ }^{1} \mathrm{H} \mathrm{NMR}\left(\mathrm{CDCl}_{3}\right.$, $400 \mathrm{MHz}) \delta$ 7.37-7.33 (m, $3 \mathrm{H}), 7.29-7.26$ (m, $2 \mathrm{H}), 6.98$ (d, $J=$ $11.8 \mathrm{~Hz}, 1 \mathrm{H}), 6.55$ (s, $1 \mathrm{H}), 5.91$ (d, $J=11.8 \mathrm{~Hz}, 1 \mathrm{H}), 3.98$ (s, 3 H), 3.51 (s, $3 \mathrm{H}), 2.68$ (t, $J=7.5 \mathrm{~Hz}, 2 \mathrm{H}$ ), 1.76 (hex, $J=7.5 \mathrm{~Hz}$, $2 \mathrm{H}), 0.97(\mathrm{t}, J=7.5 \mathrm{~Hz}, 3 \mathrm{H}) \cdot{ }^{13} \mathrm{C}$ NMR $\left(\mathrm{CDCl}_{3}, 100 \mathrm{MHz}\right) \delta$ 165.5 (s), 162.8 (s), 156.6 (s), 151.5 (s), 141.7 (d), 139.4 (s), 129.0 (d, 2 CH), 127.8 (d, 2 CH), 127.7 (d), 123.2 (d), 122.1 (s), 107.5 (d), 53.2 (q), 51.0 (q), $37.6(\mathrm{t}), 21.7$ (t), $13.9(\mathrm{q}) . \mathrm{MS}(\mathrm{m} / \mathrm{z})$ $311\left(\mathrm{M}^{+}, 6\right), 310$ ([M-1] $\left.]^{+}, 20\right), 283$ (62), 252 (100), 236 (19). HRMS (EI) calcd for $\mathrm{C}_{19} \mathrm{H}_{21} \mathrm{NO}_{3}[\mathrm{M}]^{+}, 311.1521$. Found: 311.1534.<smiles></smiles>

Pyridine 3c: $60 \%$ Yield. White solid. $\mathrm{Mp}=56-58{ }^{\circ} \mathrm{C} . \mathrm{R}_{f}=$ 0.45 (Hexane/AcOEt : 5/1). IR $v\left(\mathrm{~cm}^{-1}\right) 1727,1589 .{ }^{1} \mathrm{H}$ NMR $\left(\mathrm{CDCl}_{3}, 400 \mathrm{MHz}\right) \delta$ 7.38-7.34 (m, $\left.3 \mathrm{H}\right), 7.28-7.25$ (m, $\left.2 \mathrm{H}\right), 7.02$ $(\mathrm{d}, J=11.6 \mathrm{~Hz}, 1 \mathrm{H}), 6.53(\mathrm{~s}, 1 \mathrm{H}), 5.91(\mathrm{~d}, J=11.6 \mathrm{~Hz}, 1 \mathrm{H})$, 3.99 (s, $3 \mathrm{H}$ ), 3.50 (s, $3 \mathrm{H}$ ), 3.13 (hept, $J=6.7 \mathrm{~Hz}, 1 \mathrm{H}$ ), 1.25 (d, $J$ = $6.7 \mathrm{~Hz}, 6 \mathrm{H}) \cdot{ }^{13} \mathrm{C} \mathrm{NMR}\left(\mathrm{CDCl}_{3}, 100 \mathrm{MHz}\right) \delta 165.5$ (s), 163.0 (s), 160.9 (s), 151.5 (s), 141.7 (d), 139.6 (s), 129.0 (d, 2 CH), 127.8 (d, 2 CH), 127.6 (d), 123.4 (d), 121.1 (s), 107.6 (d), 53.1 (q), 51.0 (q), 32.7 (d), 21.7 (q, $\left.2 \mathrm{CH}_{3}\right)$. MS (m/z) $311\left(\mathrm{M}^{+}, 11\right)$, 268 (16), 252 (100), 236 (20). HRMS (EI) calcd for $\mathrm{C}_{19} \mathrm{H}_{21} \mathrm{NO}_{3}$ $[\mathrm{M}]^{+}, 311.1521$. Found: 311.1529.<smiles>COC(=O)/C=C\c1c(-c2ccccc2)cc(OC)nc1Br</smiles>

Pyridine 3d: $55 \%$ Yield. Colorless oil. $\quad \mathrm{R}_{f}=0.41$ (Hexane/AcOEt : 5/1). IR $v\left(\mathrm{~cm}^{-1}\right)$ 1727, 1585. ${ }^{1} \mathrm{H} \mathrm{NMR}\left(\mathrm{CDCl}_{3}\right.$, $400 \mathrm{MHz}) \delta 7.36$ (d, $J=11.7 \mathrm{~Hz}, 1 \mathrm{H}), 7.33-7.28$ (m, $3 \mathrm{H}), 7.18-$ 7.15 (m, $2 \mathrm{H}), 6.53$ (s, $1 \mathrm{H}), 5.74$ (d, $J=11.7 \mathrm{~Hz}, 1 \mathrm{H}), 3.97$ (s, 3 $\mathrm{H}), 3.44$ (s, $3 \mathrm{H}), 1.41$ (s, $9 \mathrm{H}) .{ }^{13} \mathrm{C} \mathrm{NMR}\left(\mathrm{CDCl}_{3}, 100 \mathrm{MHz}\right) \delta$ 165.3 (s), 161.8 (s), 161.2 (s), 153.2 (s), 144.3 (d), 140.4 (s), 129.1 (d, 2 CH), 127.7 (d, 2 CH), 127.4 (d), 122.7 (d), 122.0 (s), 108.0 (d), 53.1 (q), 51.0 (q), 39.8 (s), 30.0 (q, $\left.3 \mathrm{CH}_{3}\right) . \mathrm{MS}(\mathrm{m} / \mathrm{z})$ $325\left(\mathrm{M}^{+},<5\right), 324$ [(M-1) $\left.{ }^{+}, 19\right], 310$ (12), 268 (100), 266 (87). HRMS (EI) calcd for $\mathrm{C}_{20} \mathrm{H}_{22} \mathrm{NO}_{3}[\mathrm{M}-1]^{+}$, 324.1594. Found: 324.1590. 
<smiles>COc1cc(-c2ccccc2)c(/C=C\C(C)C)c(-c2ccccc2)n1</smiles>

Pyridine 3e: $67 \%$ Yield. White solid. $\mathrm{Mp}=82-84{ }^{\circ} \mathrm{C} . \mathrm{R}_{f}=$ 0.29 (Hexane/AcOEt : 5/1). IR $v\left(\mathrm{~cm}^{-1}\right)$ 1723, 1587. ${ }^{1} \mathrm{H}$ NMR $\left(\mathrm{CDCl}_{3}, 400 \mathrm{MHz}\right) \delta$ 7.63-7.60 (m, $\left.2 \mathrm{H}\right), 7.40-7.30$ (m, $\left.8 \mathrm{H}\right), 6.87$ $(\mathrm{d}, J=11.8 \mathrm{~Hz}, 1 \mathrm{H}), 6.71(\mathrm{~s}, 1 \mathrm{H}), 5.72(\mathrm{~d}, J=11.8 \mathrm{~Hz}, 1 \mathrm{H})$, 4.03 (s, $3 \mathrm{H}$ ), 3.38 (s, $3 \mathrm{H}) .{ }^{13} \mathrm{C} \mathrm{NMR}\left(\mathrm{CDCl}_{3}, 100 \mathrm{MHz}\right) \delta 165.6$ (s), 162.9 (s), 154.8 (s), 152.4 (s), 141.1 (d), 140.2 (s), 139.1 (d), 129.8 (d, 2 CH), 129.0 (d, 2 CH), 128.0 (d), 127.85 (d, 2 CH), 127.78 (d), 127.6 (d, 2 CH), 123.2 (d), 121.8 (s), 109.6 (d), 53.4 (q), 50.9 (q). MS (m/z) 345 (M+10), 286 (61), 97 (20), 69 (100). HRMS (EI) calcd for $\mathrm{C}_{22} \mathrm{H}_{19} \mathrm{NO}_{3}[\mathrm{M}]^{+}$, 345.1365. Found: 345.1354.

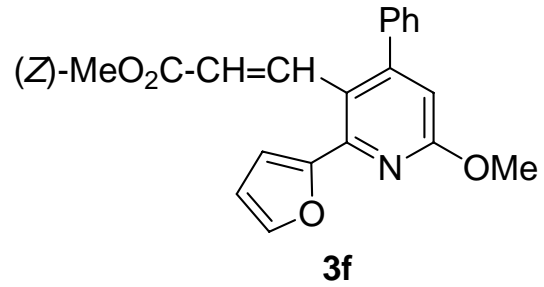

Pyridine 3f: $69 \%$ Yield. White solid. $\mathrm{Mp}=81-83^{\circ} \mathrm{C} . \mathrm{R}_{\mathrm{f}}=0.26$ (Hexane/AcOEt : 5/1). IR $v\left(\mathrm{~cm}^{-1}\right)$ 1725, 1593. ${ }^{1} \mathrm{H} \mathrm{NMR}\left(\mathrm{CDCl}_{3}\right.$, $400 \mathrm{MHz}) \delta 7.51$ (dd, $J=0.9$ and $1.8 \mathrm{~Hz}, 1 \mathrm{H}), 7.36-7.25(\mathrm{~m}, 6$ H), 7.02 (dd, $J=0.9$ and $3.4 \mathrm{~Hz}, 1 \mathrm{H}$ ), 6.63 (s, $1 \mathrm{H}$ ), 6.50 (dd, $J=$ 1.8 and $3.4 \mathrm{~Hz}, 1 \mathrm{H}), 5.84(\mathrm{~d}, J=11.7 \mathrm{~Hz}, 1 \mathrm{H}), 4.03(\mathrm{~s}, 3 \mathrm{H})$, 3.39 (s, $3 \mathrm{H}) .{ }^{13} \mathrm{C} \mathrm{NMR}\left(\mathrm{CDCl}_{3}, 100 \mathrm{MHz}\right) \delta 165.6$ (s), 162.8 (s), 153.8 (s), 153.3 (s), 143.5 (s), 143.2 (d), 141.4 (d), 139.2 (s), 129.2 (d, 2 CH), 127.8 (d, 3 CH), 122.8 (d), 120.2 (s), 111.9 (d), 111.5 (d), 110.0 (d), 53.4 (q), 51.0 (q). MS (m/z) $335\left(\mathrm{M}^{+}, 36\right)$, 318 (100), 276 (49), 204 (37). HRMS (EI) calcd for $\mathrm{C}_{20} \mathrm{H}_{17} \mathrm{NO}_{4}$ $[\mathrm{M}]^{+}$, 335.1152. Found: 335.1151.

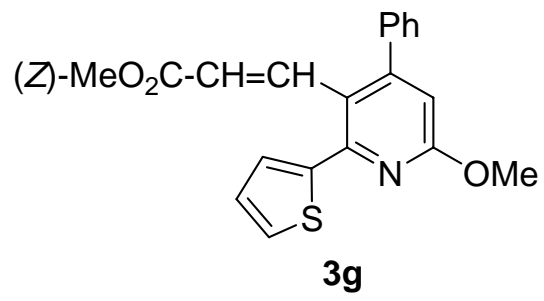

Pyridine 3g: $73 \%$ Yield. White solid. $\mathrm{Mp}=113-115{ }^{\circ} \mathrm{C} . \mathrm{R}_{\mathrm{f}}=$ 0.29 (Hexane/AcOEt : 5/1). IR $\vee\left(\mathrm{cm}^{-1}\right)$ 1725, 1584. ${ }^{1} \mathrm{H}$ NMR $\left(\mathrm{CDCl}_{3}, 400 \mathrm{MHz}\right) \delta 7.53$ (dd, $J=1.0$ and $3.7 \mathrm{~Hz}, 1 \mathrm{H}$ ), 7.42 (dd, $J=1.0$ and $5.1 \mathrm{~Hz}, 1 \mathrm{H}$ ), 7.39-7.35 (m, $3 \mathrm{H}), 7.29-7.27$ (m, $2 \mathrm{H})$, $7.18(\mathrm{~d}, J=11.7 \mathrm{~Hz}, 1 \mathrm{H}), 7.09$ (dd, $J=3.7$ and $5.1 \mathrm{~Hz}, 1 \mathrm{H})$, 6.66 (s, $1 \mathrm{H}$ ), 5.92 (d, $J=11.7 \mathrm{~Hz}, 1 \mathrm{H}$ ), 4.07 (s, $3 \mathrm{H}$ ), 3.41 (s, 3

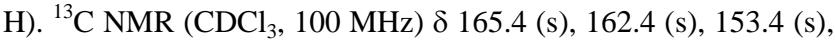
146.7 (s), 145.1 (s), 141.2 (d), 139.2 (s), 129.2 (d, 2 CH), 128.2 (d), 128.0 (d), 127.9 (d, 3 CH), 127.6 (d), 124.1 (d), 120.3 (s), 110.0 (d), 53.6 (q), 51.2 (q). MS (m/z) 351 ( $\left.\mathrm{M}^{+}, 20\right), 292$ (100), 277 (22), 260 (25). HRMS (EI) calcd for $\mathrm{C}_{20} \mathrm{H}_{17} \mathrm{NO}_{3} \mathrm{~S}[\mathrm{M}]^{+}$, 351.0924. Found: 351.0925.<smiles>C=Cc1nc(OC)cc(-c2ccccc2)c1/C=C\COC</smiles>

$3 h$

Pyridine 3h: $75 \%$ Yield. White solid. $\mathrm{Mp}=72-74{ }^{\circ} \mathrm{C} . \mathrm{R}_{f}=$ 0.41 (Hexane/AcOEt : 5/1). IR $v\left(\mathrm{~cm}^{-1}\right)$ 1727, 1582. ${ }^{1} \mathrm{H}$ NMR $\left(\mathrm{CDCl}_{3}, 400 \mathrm{MHz}\right) \delta$ 7.41-7.36 (m, $\left.3 \mathrm{H}\right), 7.32-7.29(\mathrm{~m}, 2 \mathrm{H})$, 6.92-6.85 (m, $2 \mathrm{H}), 6.64$ (s, $1 \mathrm{H}), 6.50$ (dd, $J=2.3$ and $16.8 \mathrm{~Hz}, 1$ H), $6.05(\mathrm{~d}, J=11.8 \mathrm{~Hz}, 1 \mathrm{H}), 5.44(\mathrm{dd}, J=2.3$ and $10.6 \mathrm{~Hz}, 1$ $\mathrm{H}), 4.04$ (s, $3 \mathrm{H}), 3.56$ (s, $3 \mathrm{H}) .{ }^{13} \mathrm{C} \mathrm{NMR}\left(\mathrm{CDCl}_{3}, 100 \mathrm{MHz}\right) \delta$ 165.7 (s), 162.5 (s), 151.9 (s), 149.2 (s), 140.8 (d), 138.8 (s), 133.3 (d), 128.9 (d, 2 CH), 128.0 (d, 3 CH), 124.1 (d), 122.1 (s), 119.3 (t), 110.1 (d), 53.1 (q), 51.4 (q). MS (m/z) $295\left(\mathrm{M}^{+}, 11\right)$, 236 (100), 204 (30). HRMS (EI) calcd for $\mathrm{C}_{18} \mathrm{H}_{17} \mathrm{NO}_{3}[\mathrm{M}]^{+}$, 295.1208. Found: 295.1202.<smiles>COC/C=C\c1c(-c2ccc(Cl)cc2)cc(OC)nc1OC</smiles>

Pyridine 3i: 62\% Yield. Colorless oil. $\mathrm{R}_{f}=0.29$ (Hexane/AcOEt : 5/1). IR $v\left(\mathrm{~cm}^{-1}\right)$ 1726, 1560. ${ }^{1} \mathrm{H} \mathrm{NMR}\left(\mathrm{CDCl}_{3}\right.$, $400 \mathrm{MHz}) \delta 7.33$ (d, $J=8.2 \mathrm{~Hz}, 2 \mathrm{H}), 7.21$ (d, $J=8.2 \mathrm{~Hz}, 2 \mathrm{H})$, 6.87 (d, $J=11.8 \mathrm{~Hz}, 1 \mathrm{H}), 6.51(\mathrm{~s}, 1 \mathrm{H}), 5.96$ (d, $J=11.8 \mathrm{~Hz}, 1$ $\mathrm{H}), 3.96$ (s, $3 \mathrm{H}), 3.56$ (s, $3 \mathrm{H}), 2.41$ (s, $3 \mathrm{H}) .{ }^{13} \mathrm{C} \mathrm{NMR}\left(\mathrm{CDCl}_{3}\right.$, $100 \mathrm{MHz}$ ) $\delta 165.6$ (s), 162.7 (s), 153.5 (s), 150.2 (s), 141.7 (d), 137.6 (s), 134.0 (s), 130.3 (d, 2 CH), 128.2 (d, 2 CH), 123.3 (d), 122.2 (s), 107.6 (d), 53.4 (q), 51.3 (q), 23.0 (q). MS (m/z) 317 $\left(\mathrm{M}^{+}, 45\right), 316\left([\mathrm{M}-1]^{+}, 82\right), 258$ (100). HRMS (EI) calcd for $\mathrm{C}_{17} \mathrm{H}_{15} \mathrm{ClNO}_{3}[\mathrm{M}-1]^{+}$, 316.0735. Found: 316.0732.<smiles>COC/C=C\c1c(-c2ccc(OC)cc2)cc(OC)nc1OC</smiles>

Pyridine 3j: 66\% Yield. Colorless oil. $\mathrm{R}_{f}=0.21$ (Hexane/AcOEt : 5/1). IR $v\left(\mathrm{~cm}^{-1}\right) 1726,1590,1514 .{ }^{1} \mathrm{H}$ NMR $\left(\mathrm{CDCl}_{3}, 400 \mathrm{MHz}\right) \delta 7.24(\mathrm{~d}, J=8.5 \mathrm{~Hz}, 2 \mathrm{H}), 6.91-6.86(\mathrm{~m}, 3$ H), 6.53 (s, $1 \mathrm{H}$ ), 5.99 (d, $J=11.8 \mathrm{~Hz}, 1 \mathrm{H}$ ), 3.96 (s, $3 \mathrm{H}$ ), 3.83 (s, $3 \mathrm{H}), 3.58$ (s, $3 \mathrm{H}), 2.41$ (s, $3 \mathrm{H}) \cdot{ }^{13} \mathrm{C} \mathrm{NMR}\left(\mathrm{CDCl}_{3}, 100 \mathrm{MHz}\right) \delta$ 165.9 (s), 162.8 (s), 159.5 (s), 153.3 (s), 151.2 (s), 142.4 (d), 131.6 (s), 130.3 (d, 2 CH), 123.0 (d), 122.6 (s), 113.5 (d, 2 CH), 107.5 (d), 55.3 (q), 53.4 (q), 51.3 (q), 23.0 (q). MS (m/z) $313\left(\mathrm{M}^{+}\right.$, 
51), 312 ([M-1] $\left.]^{+}, 60\right), 254$ (100), 252 (62). HRMS (EI) calcd for $\mathrm{C}_{18} \mathrm{H}_{18} \mathrm{NO}_{4}[\mathrm{M}-1]^{+}$, 312.1230. Found: 312.1230.<smiles>COC(=O)/C=C\c1c(Br)cc(OC)nc1Br</smiles>

Pyridine 3k: $71 \%$ Yield. Colorless oil. $\mathrm{R}_{f}=0.32$ (Hexane/AcOEt : 5/1). IR $v\left(\mathrm{~cm}^{-1}\right) 1731,1596 .{ }^{1} \mathrm{H} \mathrm{NMR}\left(\mathrm{CDCl}_{3}\right.$, $400 \mathrm{MHz}) \delta 7.04(\mathrm{~d}, J=11.8 \mathrm{~Hz}, 1 \mathrm{H}), 6.43(\mathrm{~s}, 1 \mathrm{H}), 6.16(\mathrm{~d}, J=$ $11.8 \mathrm{~Hz}, 1 \mathrm{H}), 3.91$ (s, $3 \mathrm{H}), 3.61$ (s, $3 \mathrm{H}), 2.45$ (t, $J=7.6 \mathrm{~Hz}, 2$ H), 2.33 (s, $3 \mathrm{H}), 1.49$ (m, $2 \mathrm{H}), 1.32$ (m, $2 \mathrm{H}), 0.90$ (t, J = $7.3 \mathrm{~Hz}$, $3 \mathrm{H}) .{ }^{13} \mathrm{C}$ NMR $\left(\mathrm{CDCl}_{3}, 100 \mathrm{MHz}\right) \delta 165.7$ (s), 162.8 (s), 152.1 (s), 151.9 (s), 142.3 (d), 123.5 (s), 123.3 (d), 106.6 (d), 53.2 (q), $51.4(\mathrm{q}), 32.9(\mathrm{t}), 31.5(\mathrm{t}), 22.9$ (q), 22.4 (t), $13.9(\mathrm{q})$. MS (m/z) $263\left(\mathrm{M}^{+}, 46\right), 262$ ([M-1] $\left.]^{+}, 51\right), 232$ (23), 204 (20), 190 (100), 160 (47). HRMS (EI) calcd for $\mathrm{C}_{15} \mathrm{H}_{20} \mathrm{NO}_{3}[\mathrm{M}-1]^{+}, 262.1438$. Found: 262.1434.<smiles>COC(=O)/C=C\c1c(Br)cc(OC)nc1C</smiles>

Pyridine 3l: 61\% Yield. Yellow solid. $\mathrm{Mp}=53-55^{\circ} \mathrm{C} . \mathrm{R}_{f}=$ 0.33 (Hexane/AcOEt : 5/1). IR $v\left(\mathrm{~cm}^{-1}\right)$ 1727, 1587. ${ }^{1} \mathrm{H}$ NMR $\left(\mathrm{CDCl}_{3}, 400 \mathrm{MHz}\right) \delta 7.35(\mathrm{~d}, J=11.8 \mathrm{~Hz}, 1 \mathrm{H}), 6.62(\mathrm{~s}, 1 \mathrm{H})$, 6.16 (d, $J=11.8 \mathrm{~Hz}, 1 \mathrm{H}), 3.91$ (s, $3 \mathrm{H}), 3.58$ (s, $3 \mathrm{H}), 2.32$ (s, 3 $\mathrm{H}), 1.29$ (s, $9 \mathrm{H}) .{ }^{13} \mathrm{C} \mathrm{NMR}\left(\mathrm{CDCl}_{3}, 100 \mathrm{MHz}\right) \delta 165.7$ (s), 162.9 (s), 158.8 (s), 152.3 (s), 145.4 (d), 123.3 (d), 104.9 (d), 53.1 (q), 51.3 (q), 36.1 (s), 30.1 (q, $\left.3 \mathrm{CH}_{3}\right), 23.6$ (q) -a signal for a C(s) around 123-124 ppm was not observed- MS (m/z) $263\left(\mathrm{M}^{+}, 33\right)$, $262\left[(\mathrm{M}-1)^{+}, 32\right), 248$ (23), 206 (100), 204 (53). HRMS (EI) calcd for $\mathrm{C}_{15} \mathrm{H}_{20} \mathrm{NO}_{3}[\mathrm{M}-1]^{+}$, 262.1438. Found: 262.1435.

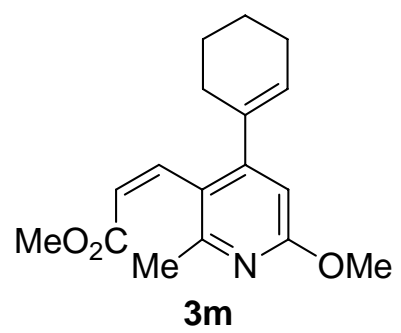

Pyridine 3m: 75\% Yield. Colorless oil. $\mathrm{R}_{f}=0.35$ (Hexane/AcOEt : 5/1). IR v ( $\left.\mathrm{cm}^{-1}\right)$ 1731, 1589. ${ }^{1} \mathrm{H} \mathrm{NMR}\left(\mathrm{CDCl}_{3}\right.$, $400 \mathrm{MHz}) \delta 6.99$ (d, $J=11.8 \mathrm{~Hz}, 1 \mathrm{H}), 6.37$ (s, $1 \mathrm{H}), 6.03$ (d, $J=$ $11.8 \mathrm{~Hz}, 1 \mathrm{H}$ ), 5.61 (bs, $1 \mathrm{H}), 3.91$ (s, $3 \mathrm{H}), 3.59$ (s, $3 \mathrm{H}), 2.35$ (s, $3 \mathrm{H}), 2.13-2.09(\mathrm{~m}, 4 \mathrm{H}), 1.68-1.60(\mathrm{~m}, 4 \mathrm{H}) .{ }^{13} \mathrm{C} \mathrm{NMR}\left(\mathrm{CDCl}_{3}\right.$, $100 \mathrm{MHz}) \delta 166.1$ (s), 162.8 (s), 154.2 (s), 152.8 (s), 142.3 (d), 136.5 (s), 128.5 (d), 122.2 (d), 122.0 (s), 105.9 (d), 53.3 (q), 51.3 (q), $28.8(\mathrm{t}), 25.3(\mathrm{t}), 22.9(\mathrm{q}), 22.8(\mathrm{t}), 21.8(\mathrm{t})$. MS (m/z) 287 $\left(\mathrm{M}^{+}, 23\right), 286\left([\mathrm{M}-1]^{+}, 16\right), 228$ (100). HRMS (EI) calcd for $\mathrm{C}_{17} \mathrm{H}_{20} \mathrm{NO}_{3}[\mathrm{M}-1]^{+}$, 286.1438. Found: 286.1434.<smiles>COC/C=C\c1c(/C(C)=C/c2ccccc2)cc(OC)nc1Br</smiles>

Pyridine 3n: 64\% Yield. Yellow oil. $\mathrm{R}_{f}=0.32$ (Hexane/AcOEt : 5/1). IR $v\left(\mathrm{~cm}^{-1}\right) 1728,1588 .{ }^{1} \mathrm{H} \mathrm{NMR}\left(\mathrm{CDCl}_{3}, 400 \mathrm{MHz}\right) \delta$ 7.40-7.36 (m, $2 \mathrm{H}), 7.32-7.25$ (m, $3 \mathrm{H}), 7.08$ (d, $J=11.7 \mathrm{~Hz}, 1$ H), $6.53(\mathrm{~s}, 1 \mathrm{H}), 6.42(\mathrm{~s}, 1 \mathrm{H}), 6.09$ (d, $J=11.7 \mathrm{~Hz}, 1 \mathrm{H}), 3.96$ (s, $3 \mathrm{H}), 3.59$ (s, $3 \mathrm{H}), 2.41$ (s, $3 \mathrm{H}), 2.10$ (s, $3 \mathrm{H}) .{ }^{13} \mathrm{C} \mathrm{NMR}\left(\mathrm{CDCl}_{3}\right.$, $100 \mathrm{MHz}$ ) $\delta 166.0$ (s), 162.9 (s), 155.5 (s), 153.1 (s), 142.1 (d), 137.3 (s), 136.4 (s), 131.0 (d), 128.9 (d, 2 CH), 128.3 (d, 2 CH), 126.9 (d), 122.8 (d), 121.9 (s), 106.1 (d), 53.4 (q), 51.4 (q), 23.0 (q), 19.0 (q). MS (m/z) $323\left(\mathrm{M}^{+}, 18\right), 322$ ([M-1] $\left.]^{+}, 7\right), 264$ (100). HRMS (EI) calcd for $\mathrm{C}_{20} \mathrm{H}_{21} \mathrm{NO}_{3}[\mathrm{M}]^{+}$, 323.1516. Found: 323.1508.<smiles>C=Cc1nc(OCC)cc(C#Cc2ccccc2)c1/C=C\COC</smiles>

$8 a$

Pyridine 8a: 65\% Yield. Yellow oil. $\mathrm{R}_{f}=0.35$ (Hexane/AcOEt : 5/1). IR $\vee\left(\mathrm{cm}^{-1}\right) 1731,1573 .{ }^{1} \mathrm{H} \mathrm{NMR}\left(\mathrm{CDCl}_{3}, 400 \mathrm{MHz}\right) \delta$ 7.52-7.50 (m, $2 \mathrm{H}), 7.38-7.36$ (m, $3 \mathrm{H}), 7.15$ (d, $J=11.8 \mathrm{~Hz}, 1$ H), 6.86-6.79 (m, $2 \mathrm{H}), 6.44$ (dd, $J=2.2$ and $16.9 \mathrm{~Hz}, 1 \mathrm{H}), 6.28$ (d, $J=11.8 \mathrm{~Hz}, 1 \mathrm{H}$ ), 5.46 (dd, $J=2.2$ and $10.5 \mathrm{~Hz}, 1 \mathrm{H}$ ), 4.46 (q, $J=7.1 \mathrm{~Hz}, 2 \mathrm{H}$ ), 3.59 (s, $3 \mathrm{H}), 1.43$ (t, $J=7.1 \mathrm{~Hz}, 3 \mathrm{H}) .{ }^{13} \mathrm{C} \mathrm{NMR}$ $\left(\mathrm{CDCl}_{3}, 100 \mathrm{MHz}\right) \delta 165.9$ (s), 162.3 (s), 149.5 (s), 139.3 (d), 133.2 (d), 133.1 (s), 131.9 (d, 2 CH), 129.1 (d), 128.4 (d, 2 CH), 124.3 (d), 124.0 (s), 122.3 (s), 119.8 (t), 112.3 (d), 95.8 (s), 86.2 (s), 61.8 (t), 51.5 (q), 14.6 (q). MS (m/z) $333\left(\mathrm{M}^{+}, 53\right), 318$ (31), 274 (18), 105 (92), 84 (100). HRMS (EI) calcd for $\mathrm{C}_{21} \mathrm{H}_{19} \mathrm{NO}_{3}$ $[\mathrm{M}]^{+}$, 333.1359. Found: 333.1360.<smiles>CCOc1cc(C#Cc2ccccc2)c(/C=C\COC)c(-c2cccs2)n1</smiles>

8b

Pyridine 8b: 66\% Yield. Yellow oil. $\mathrm{R}_{f}=0.33$ (Hexane/AcOEt : 5/1). IR $\vee\left(\mathrm{cm}^{-1}\right) 1728,1574 .{ }^{1} \mathrm{H}$ NMR $\left(\mathrm{CDCl}_{3}, 400 \mathrm{MHz}\right) \delta$ 7.54-7.52 (m, $3 \mathrm{H}$ ), 7.45 (dd, $J=1.0$ and $5.1 \mathrm{~Hz}, 1 \mathrm{H}$ ), 7.39-7.37 (m, 3 H), 7.13-7.10 (m, 2 H), 6.85 (s, 1 H), 6.35 (d, $J=11.7$ Hz, 1 
H), 4.49 (q, $J=7.1 \mathrm{~Hz}, 2 \mathrm{H}), 3.59$ (s, $3 \mathrm{H}), 1.46$ (t, $J=7.1 \mathrm{~Hz}, 3$ H). ${ }^{13} \mathrm{C} \mathrm{NMR}\left(\mathrm{CDCl}_{3}, 100 \mathrm{MHz}\right) \delta 166.4$ (s), 162.1 (s), 147.0 (s), 144.8 (s), 139.8 (d), 134.0 (s), 131.9 (d, 2 CH), 129.1 (d), 128.8 (d), 128.4 (d, 3 CH), 127.8 (d), 124.2 (d), 122.3 (s), 122.1 (s), 111.9 (d), 95.3 (s), 86.6 (s), 62.2 (t), 51.4 (q), 14.6 (q). MS (m/z) 389 (M+, 75), 374 (29), 330 (30), 302 (60), 105 (100). HRMS (EI) calcd for $\mathrm{C}_{23} \mathrm{H}_{19} \mathrm{NO}_{3} \mathrm{~S}[\mathrm{M}]^{+}$, 389.1080. Found: 389.1082.

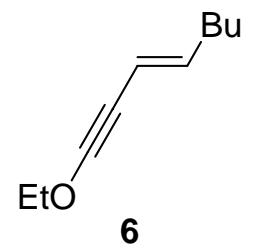

Synthesis of (E)-1-ethoxyoct-3-en-1-yne 6. Prepared as previously reported for other electron-rich enynes: ${ }^{4}$ To a solution of tetrakis(triphenylphosphine) palladium (500 mg, $0.558 \mathrm{mmol}$ ) in isopropyl amine was added (E)-l-iodo-1-hexene (1.80 g, 8.56 mmol), ethoxyacetylene (1.5 equiv., $2.57 \mathrm{~mL}$ of a solution $5 \mathrm{M}$ in hexane, $12.84 \mathrm{mmol}$ ), and a solution of copper (I) iodide (163 mg, $1.12 \mathrm{mmol})$ in isopropylamine $(5 \mathrm{~mL})$. The resulting orange solution was stirred in the dark for $6 \mathrm{~h}$. The reaction was diluted with hexane and thoroughly washed with ammonium chloride (sat. aq.). The aqueous layers were combined and extracted with hexane. The combined organic layers were dried over sodium sulfate, concentrated, and purified by flash chromatography (100\% hexane, $\mathrm{Et}_{3} \mathrm{~N}$ pretreated silica) to afford a 35\% yield of $\mathbf{6}$. Colorless oil. $\mathrm{R}_{f}=0.23$ (Hexane). ${ }^{1} \mathrm{H}$ NMR $\left(\mathrm{C}_{6} \mathrm{D}_{6}, 400 \mathrm{MHz}\right) \delta$ 6.17 (m, $1 \mathrm{H}), 5.72$ (m, $1 \mathrm{H}), 3.72$ (q, $J=7.1 \mathrm{~Hz}, 2 \mathrm{H}), 2.02$ (m, 2 H), 1.29 (m, $4 \mathrm{H}), 1.02$ (t, $J=7.1 \mathrm{~Hz}, 3 \mathrm{H}), 0.90$ (m, $J=7.1 \mathrm{~Hz}, 3$ H). ${ }^{13} \mathrm{C}$ NMR $\left(\mathrm{C}_{6} \mathrm{D}_{6}, 100 \mathrm{MHz}\right) \delta 140.9$ (d), 109.9 (d), 97.8 (s), $74.1(\mathrm{t}), 39.0(\mathrm{~s}), 32.7$ (t), $31.3(\mathrm{t}), 22.2$ (t), 14.0 (q), 13.8 (q).

\footnotetext{
${ }^{4}$ Dussault, P. H.; Han, Q.; Sloss, D. G.; Symonsbergen, D. J. Tetrahedron 1999, 55, 11437-11454.
} 
Tables with 2D-NMR (HSQC, COSY, HMBC and NOESY) experiments for 2a (400 MHz) and for 3k (300 MHz)

\begin{tabular}{|c|c|c|c|c|c|c|c|}
\hline Site & ${ }^{13} \mathrm{C}-\mathrm{NMR}(\mathrm{ppm})$ & DEPT & ${ }^{1} \mathrm{H}-\mathrm{NMR}$ & COSY & HMBC & \multicolumn{2}{|l|}{$\begin{array}{l}\text { NOESY } \\
\text { (selected) }\end{array}$} \\
\hline 1 & 49.31 & $\mathrm{C}$ & & & & & \\
\hline 2 & 49.28 & $\mathrm{CH}$ & $\begin{array}{l}3.79(\mathrm{~d}, J=7.0 \mathrm{~Hz} \\
1 \mathrm{H})\end{array}$ & 5.85 & $\begin{array}{l}49.31,121.8,131.0 \\
132.6,150.6,170.7\end{array}$ & \multicolumn{2}{|c|}{$\begin{array}{l}5.85,7.45 \\
7.32-7.13\end{array}$} \\
\hline 3 & 121.8 & $\mathrm{CH}$ & $5.85(\mathrm{~m}, 1 \mathrm{H})$ & $3.79,6.32$ & $49.31 / 49.28,117.7$ & $3.79,6.32$ & \\
\hline 4 & 131.0 & $\mathrm{CH}$ & $\begin{array}{l}6.32(\mathrm{~d}, J=8.0 \mathrm{~Hz} \\
1 \mathrm{H})\end{array}$ & 5.85 & $49.28,132.6$ & $\begin{array}{l}5.85 \\
7.13\end{array}$ & 7.32- \\
\hline 5 & 117.7 & C & & & & & \\
\hline 6 & 132.6 & C & & & & & \\
\hline 7 & 131.5 & C & & & & & \\
\hline 8 & 149.6 & C & & & & & \\
\hline 9 & 59.3 & $\mathrm{CH}_{3}$ & $4.14(\mathrm{~s}, 3 \mathrm{H})$ & & 149.6 & 3.25 & \\
\hline 10 & 61.2 & $\mathrm{CH}_{3}$ & $3.25(\mathrm{~s}, 3 \mathrm{H})$ & & 131.5 & $\begin{array}{l}4.14 \\
7.13\end{array}$ & 7.32- \\
\hline 11 & 170.7 & C & & & & & \\
\hline 12 & 51.7 & $\mathrm{CH}_{3}$ & $3.64(\mathrm{~s}, 3 \mathrm{H})$ & & 170.7 & & \\
\hline 13 & 150.6 & C & & & & & \\
\hline 14 & 127.9 & $\mathrm{CH}$ & 7.32-7.13 (m,1 H) & & & & \\
\hline 15 & 140.3 & $\mathrm{CH}$ & $7.45(\mathrm{~m}, 1 \mathrm{H})$ & 5.80 & $\begin{array}{l}\text { 166.7, } 150.6,141.8 \\
49.31\end{array}$ & $\begin{array}{l}3.79,5.80 \\
7.32-7.13\end{array}$ & \\
\hline 16 & 117.4 & $\mathrm{CH}$ & $5.80(\mathrm{~m}, 1 \mathrm{H})$ & 7.45 & $127.9,166.7$ & 7.45 & \\
\hline 17 & 166.7 & C & & & & & \\
\hline 18 & 51.1 & $\mathrm{CH}_{3}$ & $3.71(\mathrm{~s}, 3 \mathrm{H})$ & & 166.7 & & \\
\hline 19 & 141.8 & C & & & & & \\
\hline 20 & $\begin{array}{l}126.6 / 126.8 / 127.5 / 128 \\
0\end{array}$ & $\mathrm{CH}$ & $\begin{array}{l}\text { 7.32-7.13/7.46-7.39 } \\
(\mathrm{m}, 2 \mathrm{H})\end{array}$ & & & & \\
\hline 21 & $\begin{array}{l}\text { 126.6/126.8/127.5/128. } \\
0\end{array}$ & $\mathrm{CH}$ & $\begin{array}{l}7.32-7.13 / 7.46-7.39 \\
(\mathrm{~m}, 2 \mathrm{H})\end{array}$ & & & & \\
\hline 22 & $126.3 / 127.0$ & $\mathrm{CH}$ & 7.46-7.39 (m, $1 \mathrm{H})$ & & & & \\
\hline 23 & 138.0 .0 & C & & & & & \\
\hline 24 & $\begin{array}{l}\text { 126.6/126.8/127.5/128. } \\
0\end{array}$ & $\mathrm{CH}$ & $\begin{array}{l}\text { 7.32-7.13/7.46-7.39 } \\
(\mathrm{m}, 2 \mathrm{H})\end{array}$ & & & & \\
\hline 25 & $\begin{array}{l}\text { 126.6/126.8/127.5/128. } \\
0\end{array}$ & $\mathrm{CH}$ & $\begin{array}{l}7.32-7.13 / 7.46-7.39 \\
(\mathrm{~m}, 2 \mathrm{H})\end{array}$ & & & & \\
\hline 26 & $126.3 / 127.0$ & $\mathrm{CH}$ & 7.46-7.39 (m, $1 \mathrm{H})$ & & & & \\
\hline
\end{tabular}




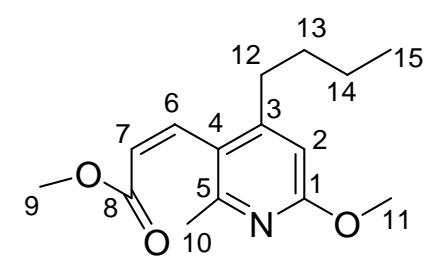

\begin{tabular}{|c|c|c|c|c|c|c|}
\hline Site & $\begin{array}{l}{ }^{13} \mathrm{C}-\mathrm{NMR} \\
(\mathrm{ppm})\end{array}$ & DEPT & ${ }^{1} \mathrm{H}-\mathrm{NMR}$ & COSY & HMBC & $\begin{array}{l}\text { NOESY } \\
\text { (selected) }\end{array}$ \\
\hline 1 & 162.8 & C & & & & \\
\hline 2 & 106.6 & $\mathrm{CH}$ & $6.43(\mathrm{~s}, 1 \mathrm{H})$ & 2.45 & $32.9,123.5,162.8$ & $\begin{array}{l}1.32,1.49,2.45 \text {, } \\
3.91\end{array}$ \\
\hline 3 & 151.9 & C & & & & \\
\hline 4 & 123.5 & C & & & & \\
\hline 5 & 152.1 & C & & & & \\
\hline 6 & 142.3 & $\mathrm{CH}$ & $7.04(\mathrm{~d}, J=11.8 \mathrm{~Hz}, 1 \mathrm{H})$ & & $151.9,152.1,165.7$ & $1.49,2.33,2.45$ \\
\hline 7 & 123.3 & $\mathrm{CH}$ & $6.16(\mathrm{~d}, J=11.8 \mathrm{~Hz}, 1 \mathrm{H})$ & & $151.9,152.1,165.7$ & \\
\hline 8 & 165.7 & C & & & & \\
\hline 9 & 51.4 & $\mathrm{CH}_{3}$ & $3.61(\mathrm{~s}, 3 \mathrm{H})$ & & 165.7 & \\
\hline 10 & 22.9 & $\mathrm{CH}_{3}$ & $2.33(\mathrm{~s}, 3 \mathrm{H})$ & & $123.5,152.1$ & \\
\hline 11 & 53.2 & $\mathrm{CH}_{3}$ & $3.91(\mathrm{~s}, 3 \mathrm{H})$ & & 162.8 & \\
\hline 12 & 32.9 & $\mathrm{CH}_{2}$ & $2.45(\mathrm{t}, \mathrm{J}=7.6 \mathrm{~Hz}, 2 \mathrm{H})$ & $1.49,6.43$ & $\begin{array}{l}22.4,31.5,106.6 \\
123.5,151.9\end{array}$ & \\
\hline 13 & 31.5 & $\mathrm{CH}_{2}$ & $1.49(\mathrm{~m}, 2 \mathrm{H})$ & $1.32,2.45$ & $13.9,22.4,32.9,151.9$ & \\
\hline 14 & 22.4 & $\mathrm{CH}_{2}$ & $1.32(\mathrm{~m}, 2 \mathrm{H})$ & $0.90,1.49$ & $13.9,31.5,32.9$ & \\
\hline 15 & 13.9 & $\mathrm{CH}_{3}$ & $0.90(\mathrm{t}, \mathrm{J}=7.3 \mathrm{~Hz}, 3 \mathrm{H})$ & 1.32 & $22.4,31.5$ & \\
\hline
\end{tabular}




\section{${ }^{1} \mathrm{H}$ and ${ }^{13} \mathrm{C}$ NMR}




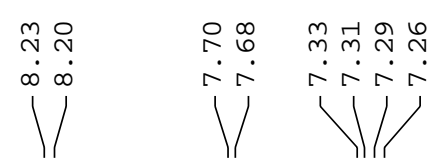

No
$\infty$
10
10

$\stackrel{\infty}{\stackrel{0}{\circ}} \stackrel{\varphi}{\dot{m}}$
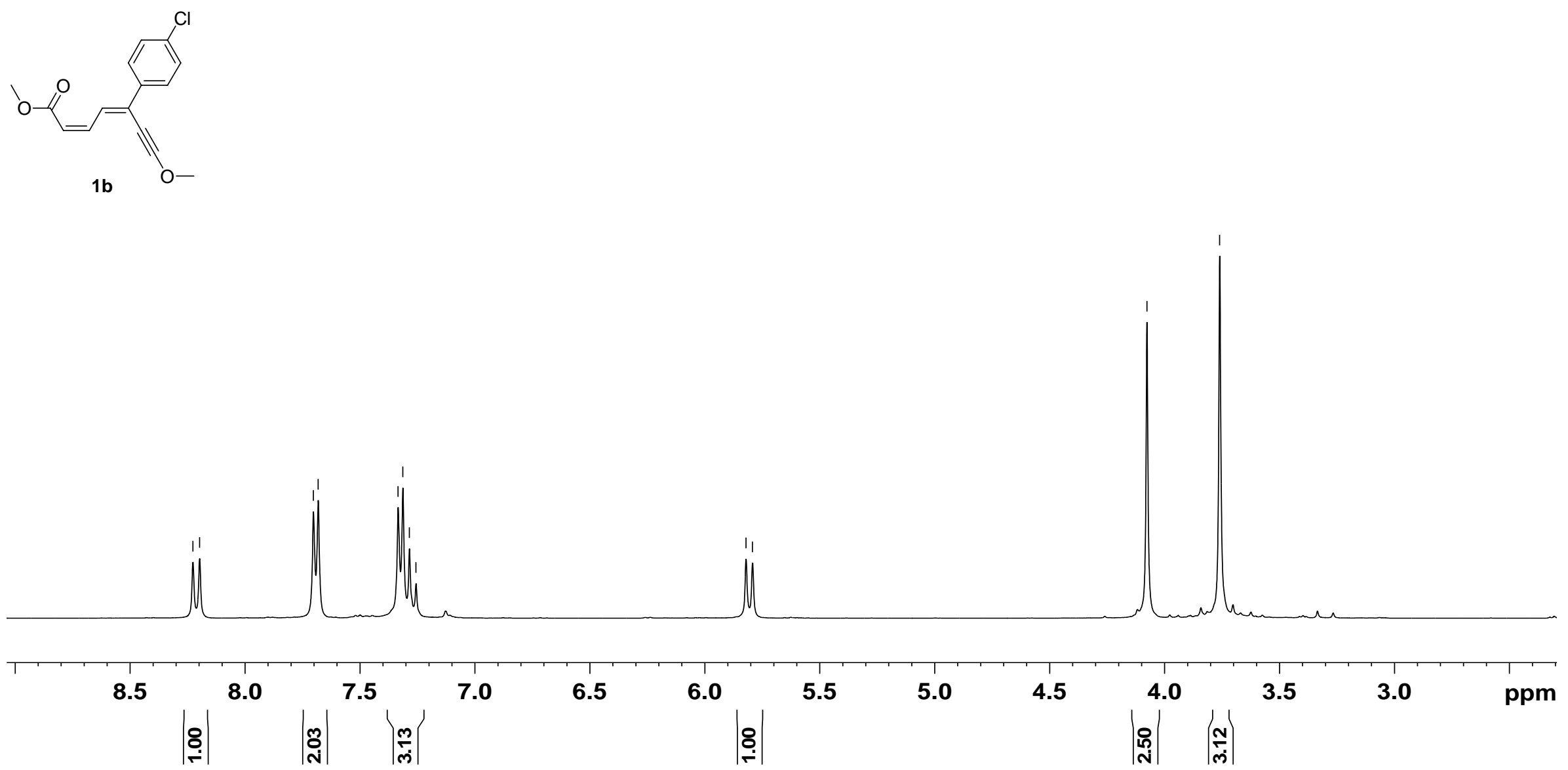

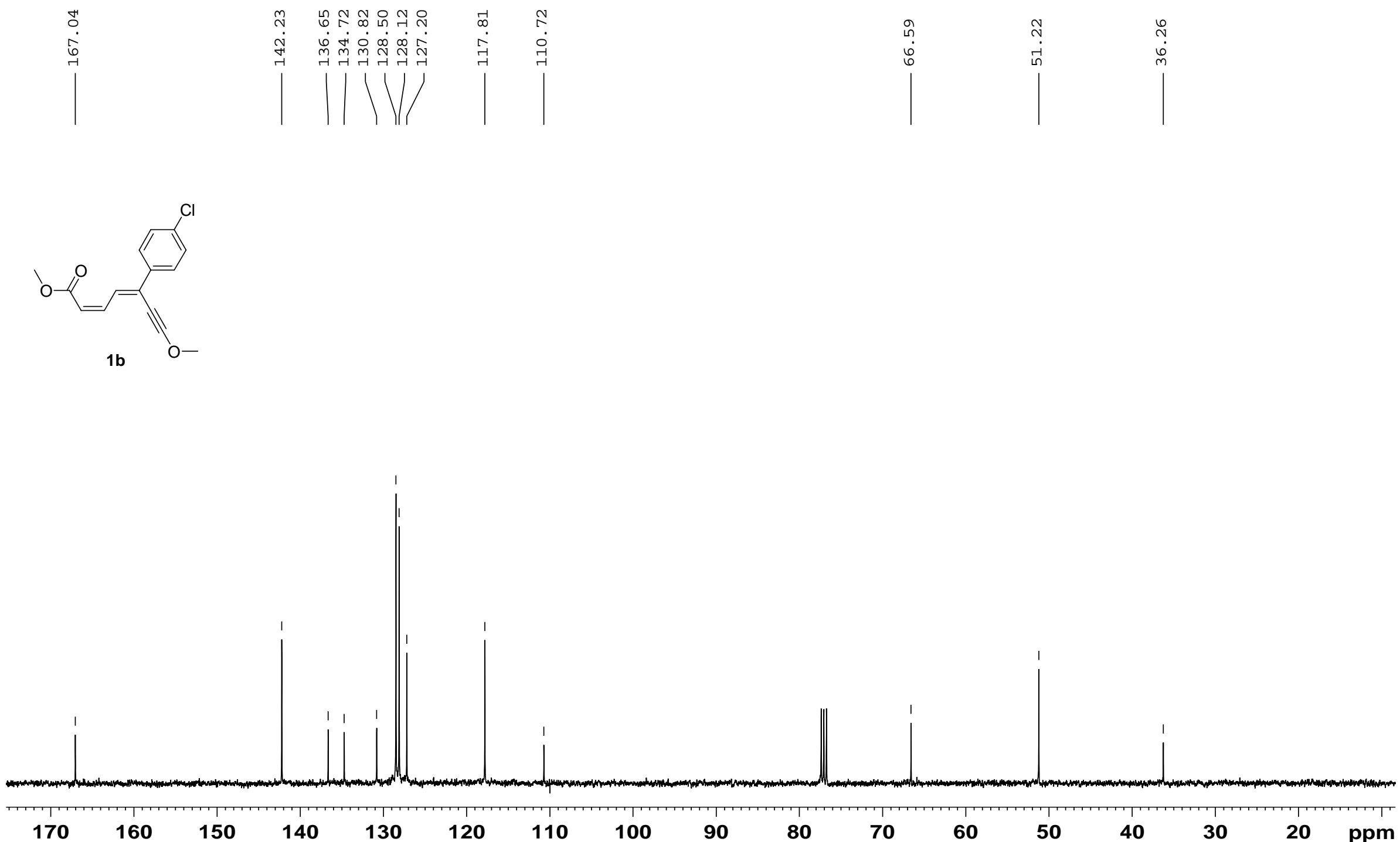


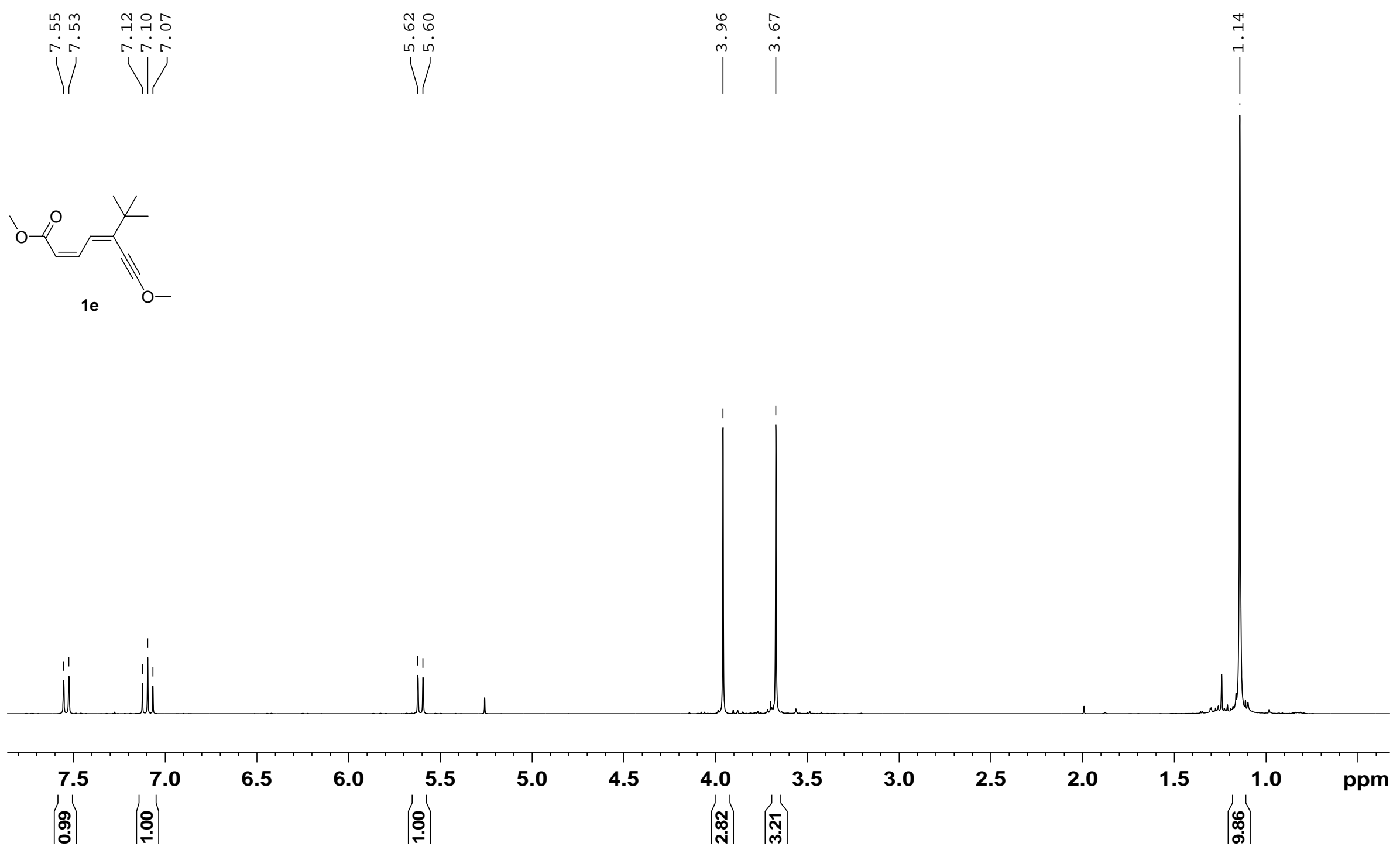



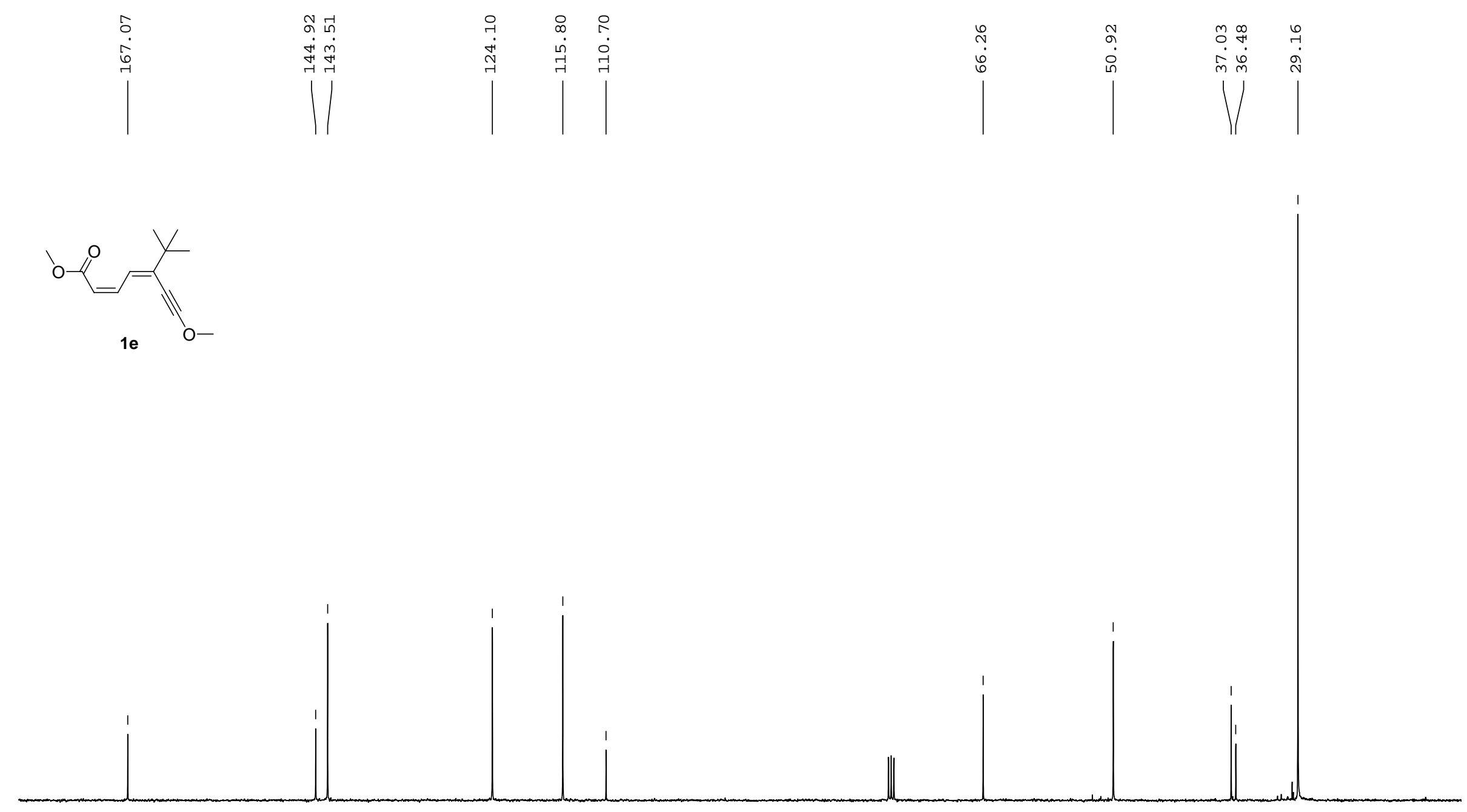

$\begin{array}{llllllllllllllllllll}170 & 160 & 150 & 140 & 130 & 120 & 110 & 100 & 90 & 80 & 70 & 60 & 50 & 40 & 30 & 20 & \text { ppm }\end{array}$ 
감ㅇำ

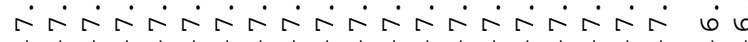

minlll

$\widehat{\infty}$

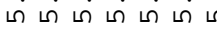

1

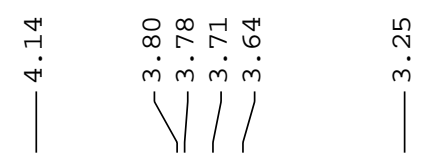
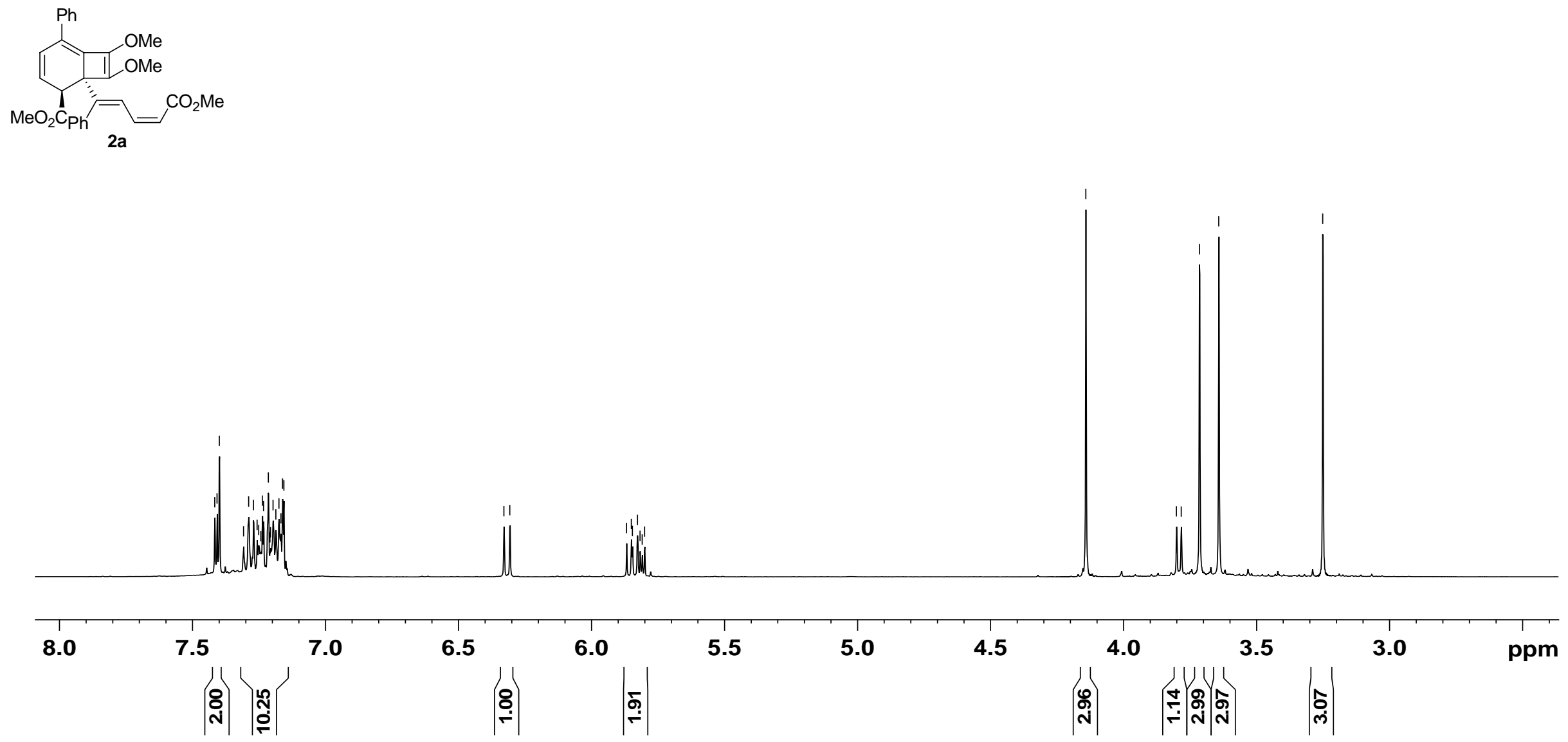


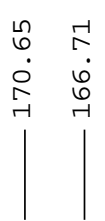

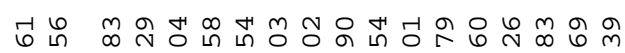

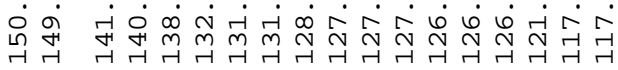

|

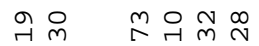

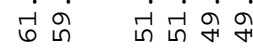

IIV
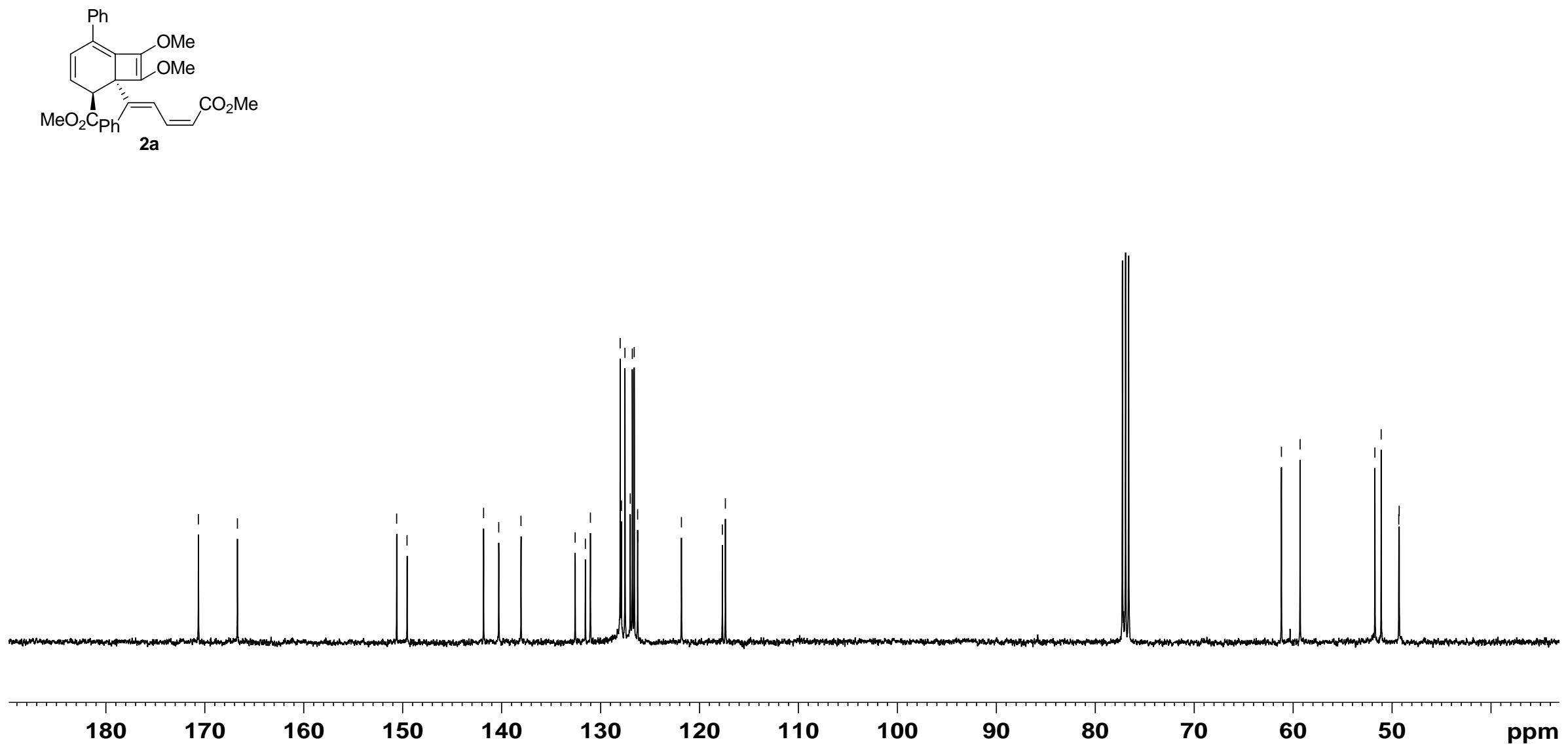


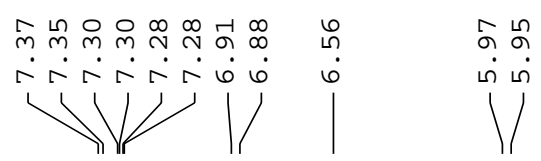

aे
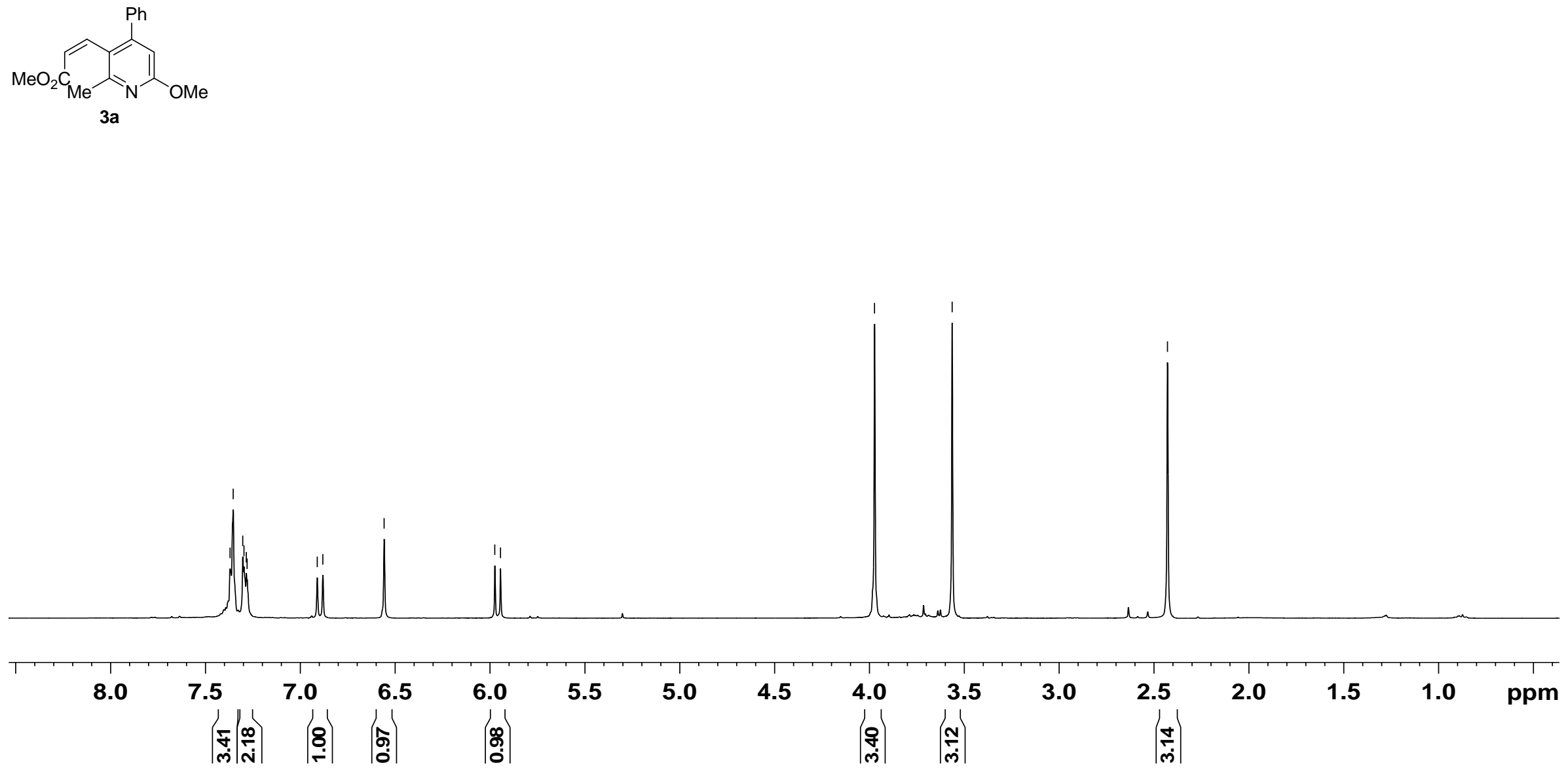

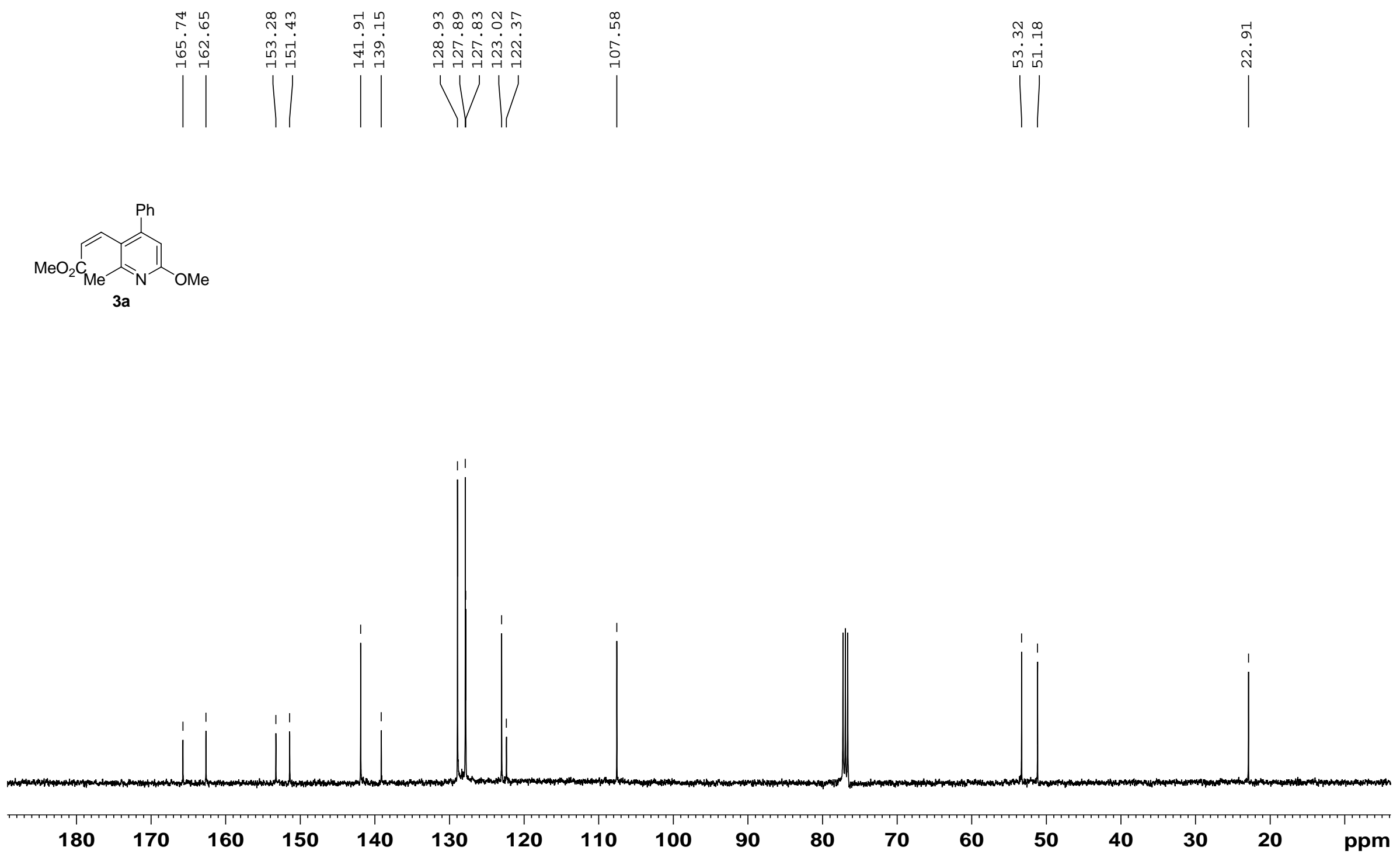


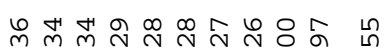

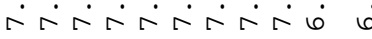

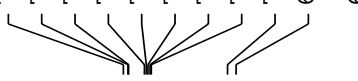

10

1

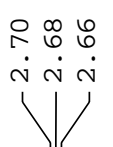

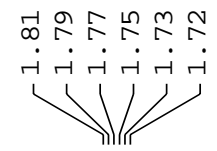

ㅇํํㅇํำ

(9

11
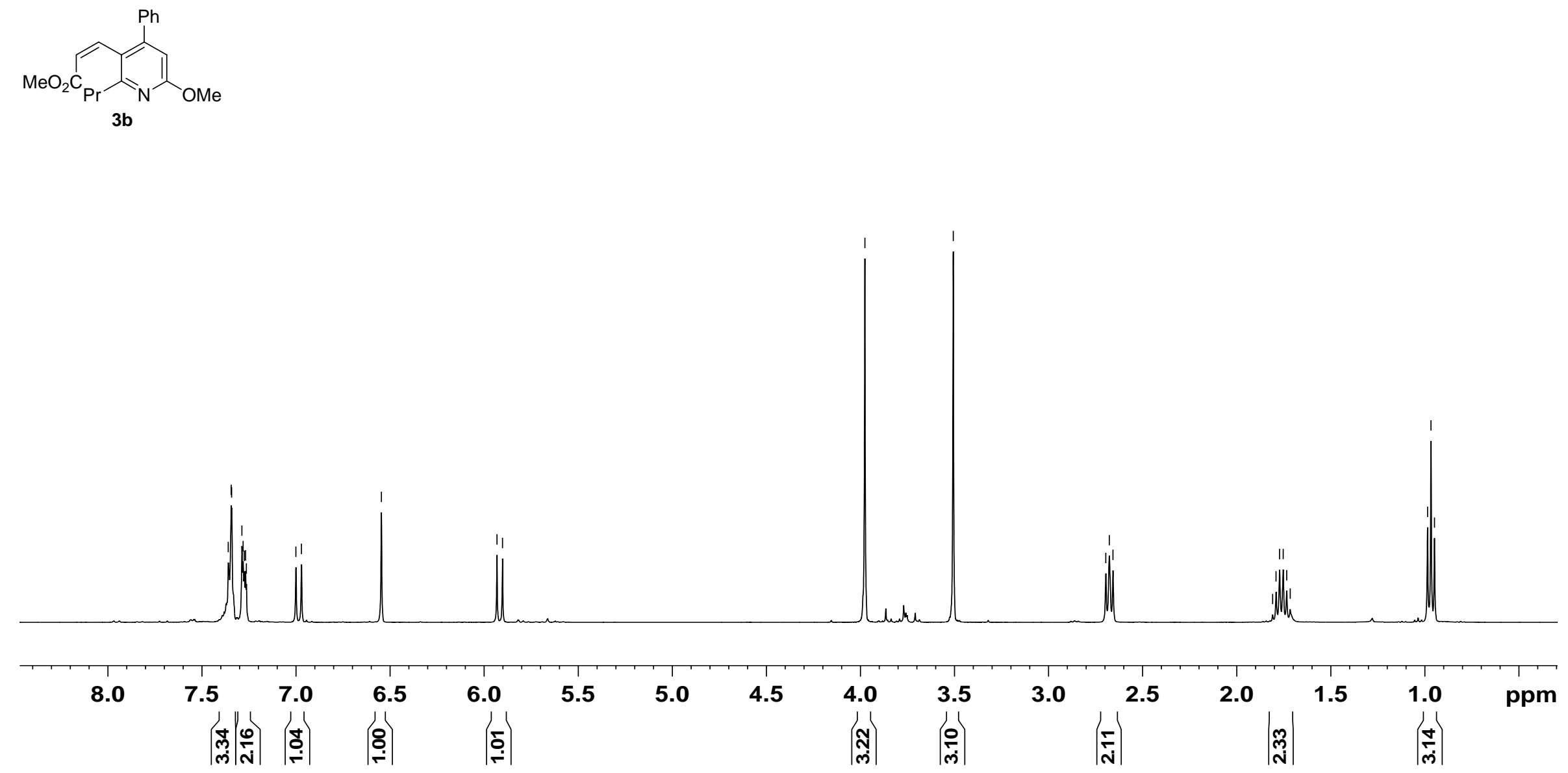


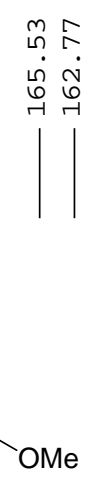

3b

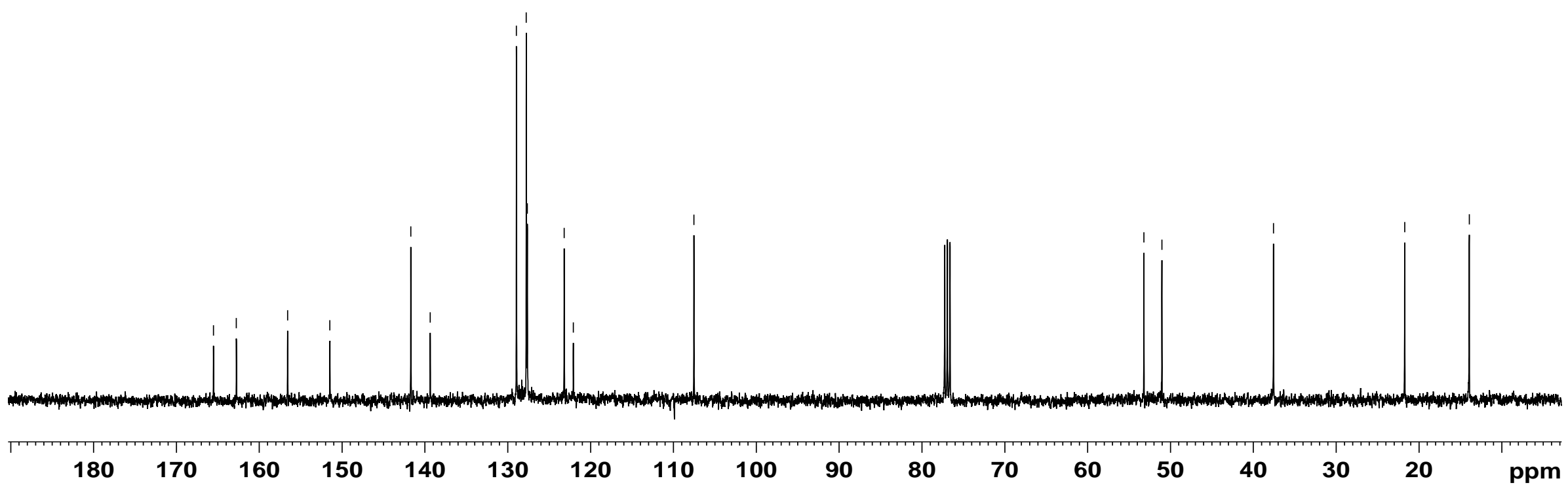




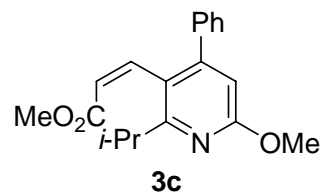

3c

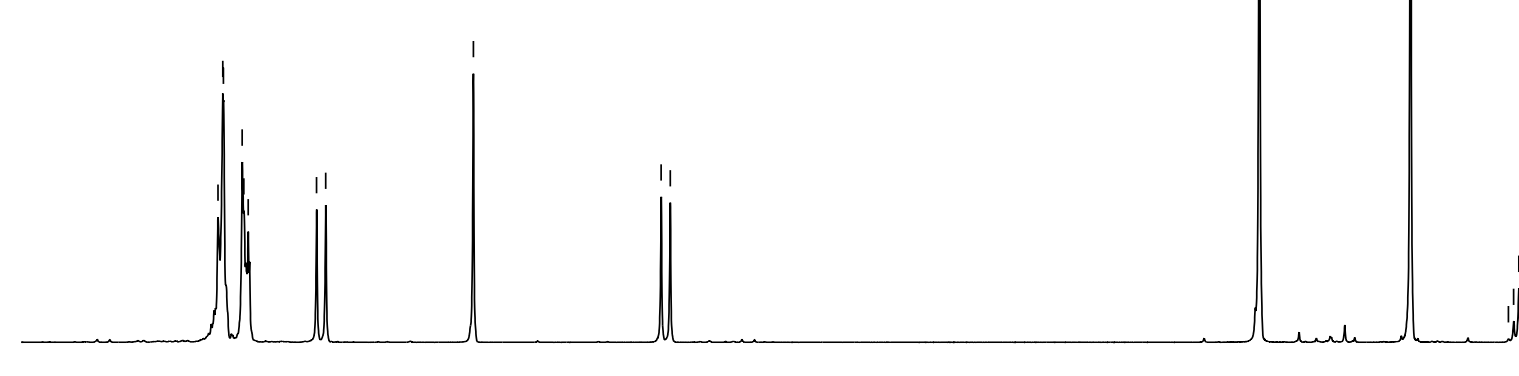

$7.5 \quad 7.0$

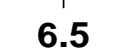

6.0

5.5

5.

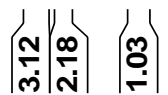

$\left|\begin{array}{l}6 \\ 8 \\ i\end{array}\right|$

$\left|\begin{array}{l}0 \\ 8 \\ i\end{array}\right|$

(్ి

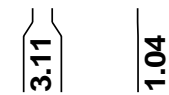

1.0 ppm

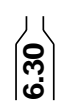



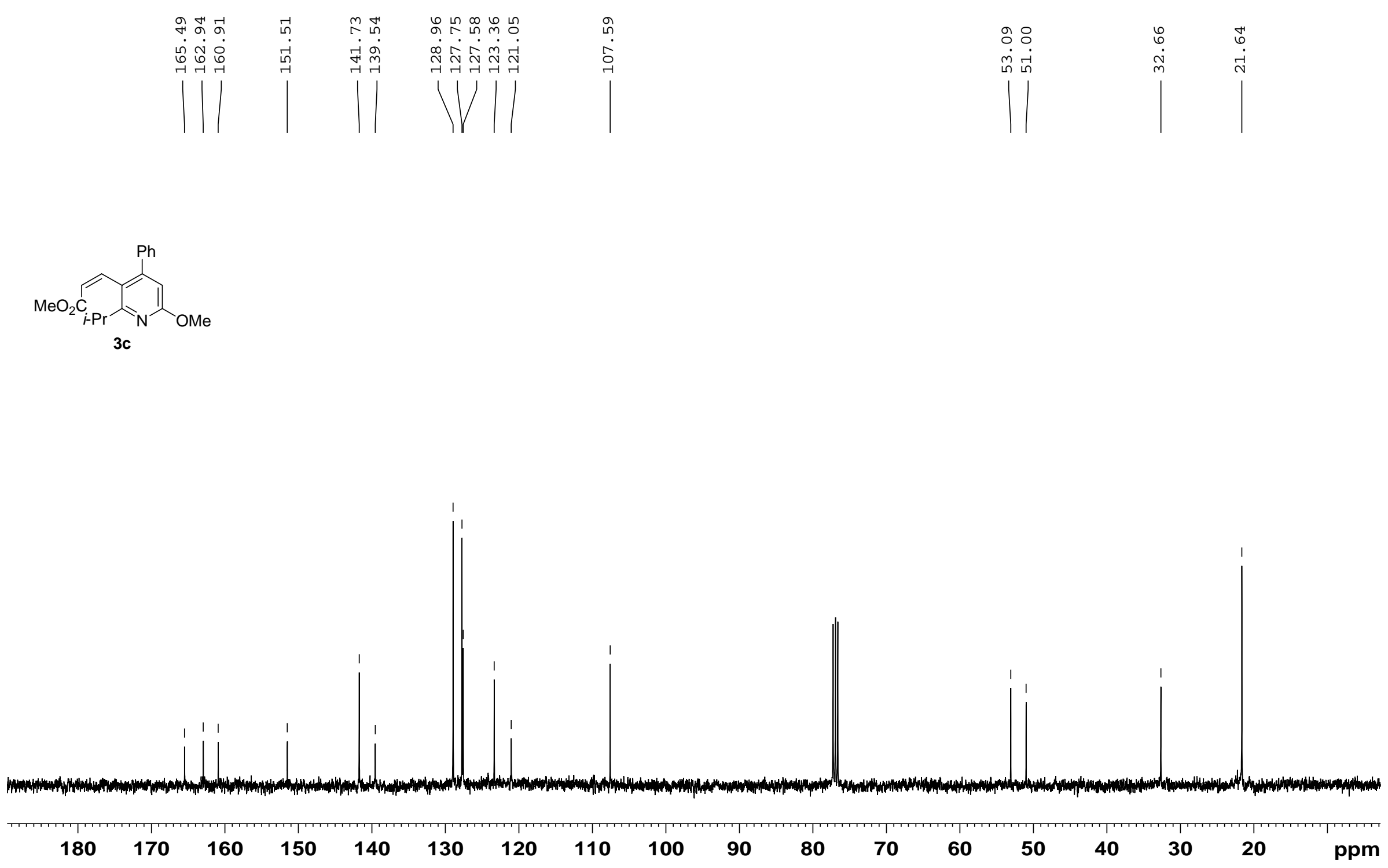


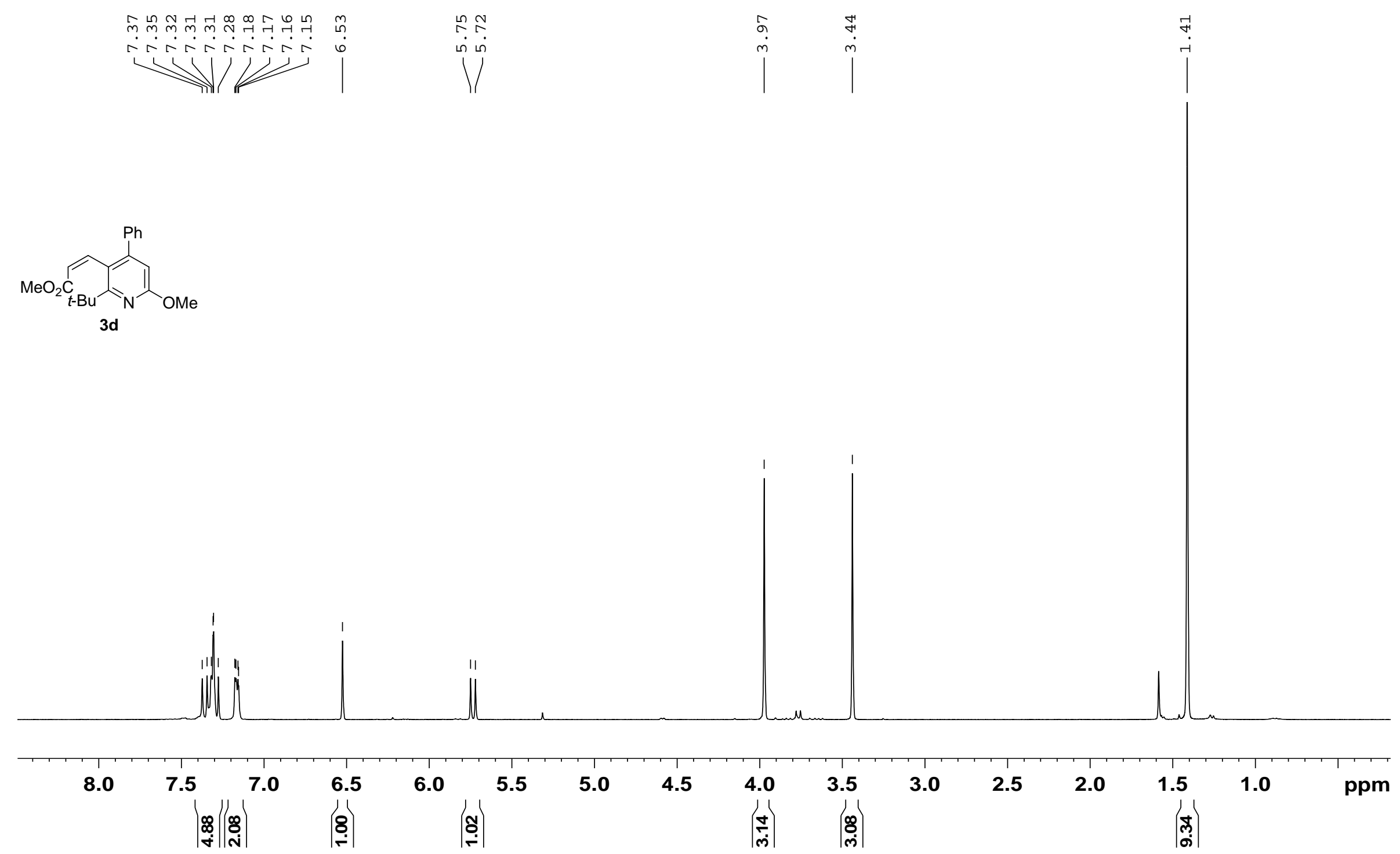




m
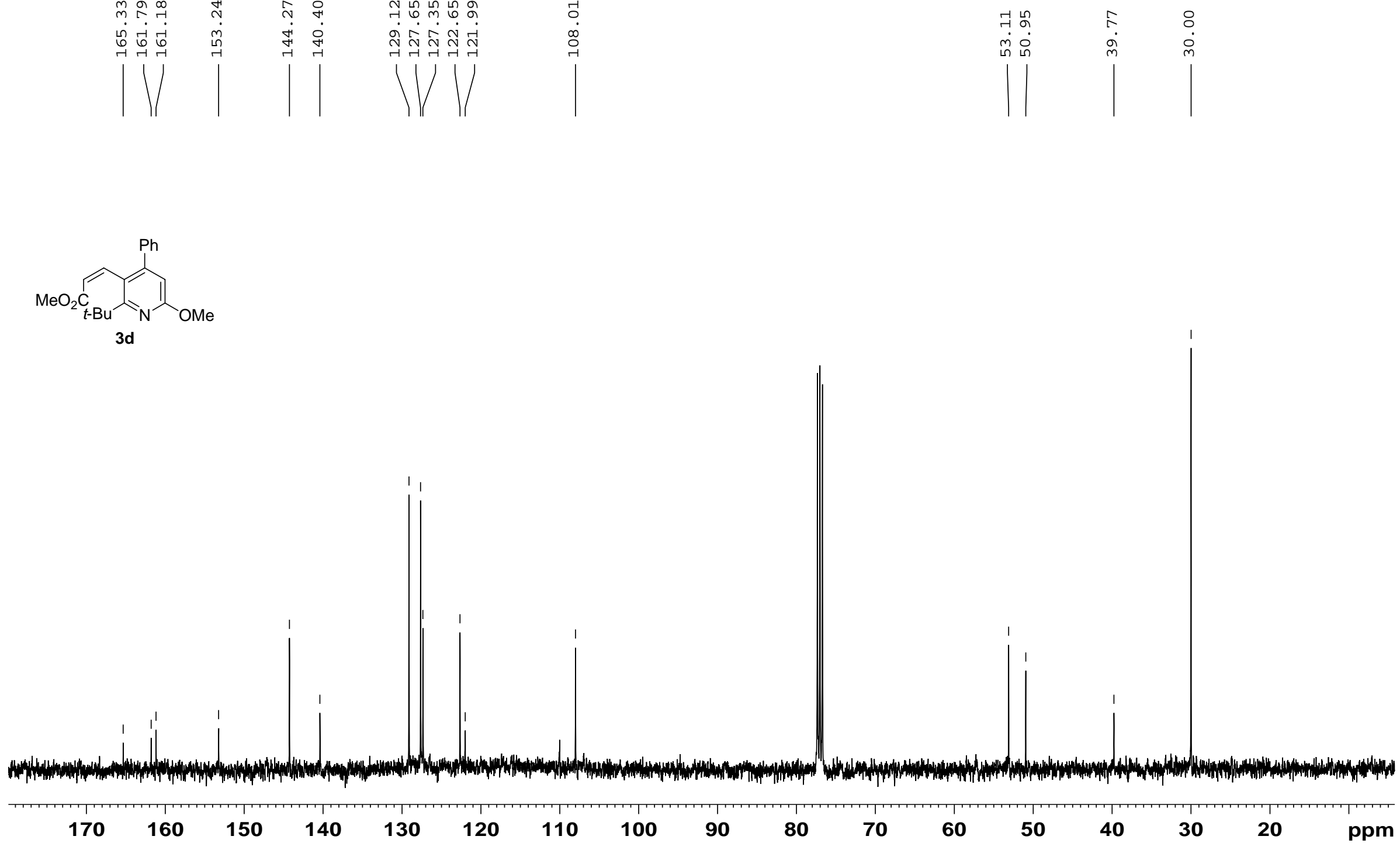


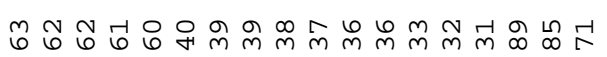

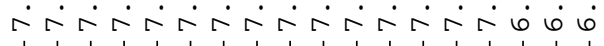

$\longrightarrow$ $\sqrt[5]{5}$

ก เ

1

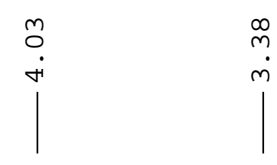

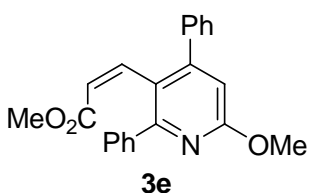

$3 e$

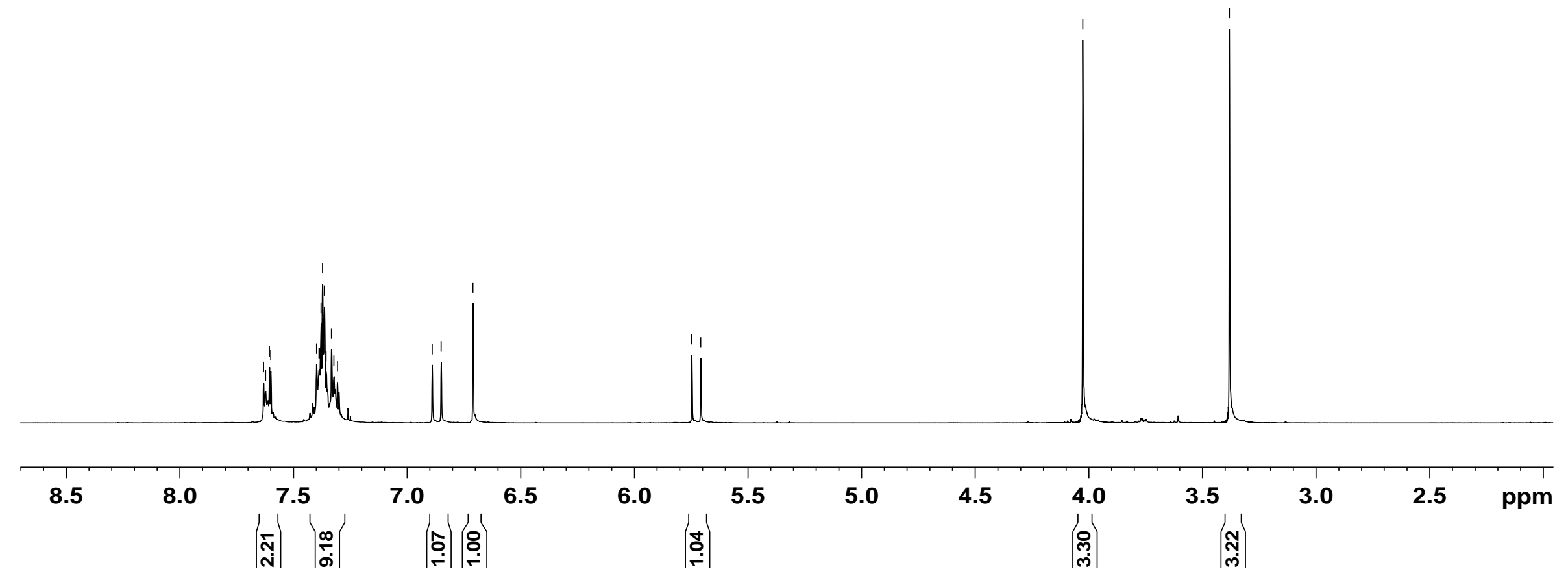




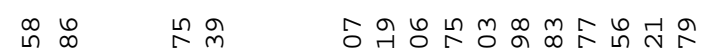

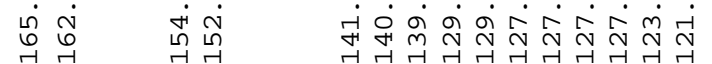

| |

11111

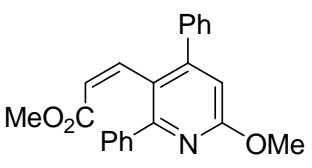

$3 e$ $\infty$

๑

ले ஓ

ณூ

$\mid$

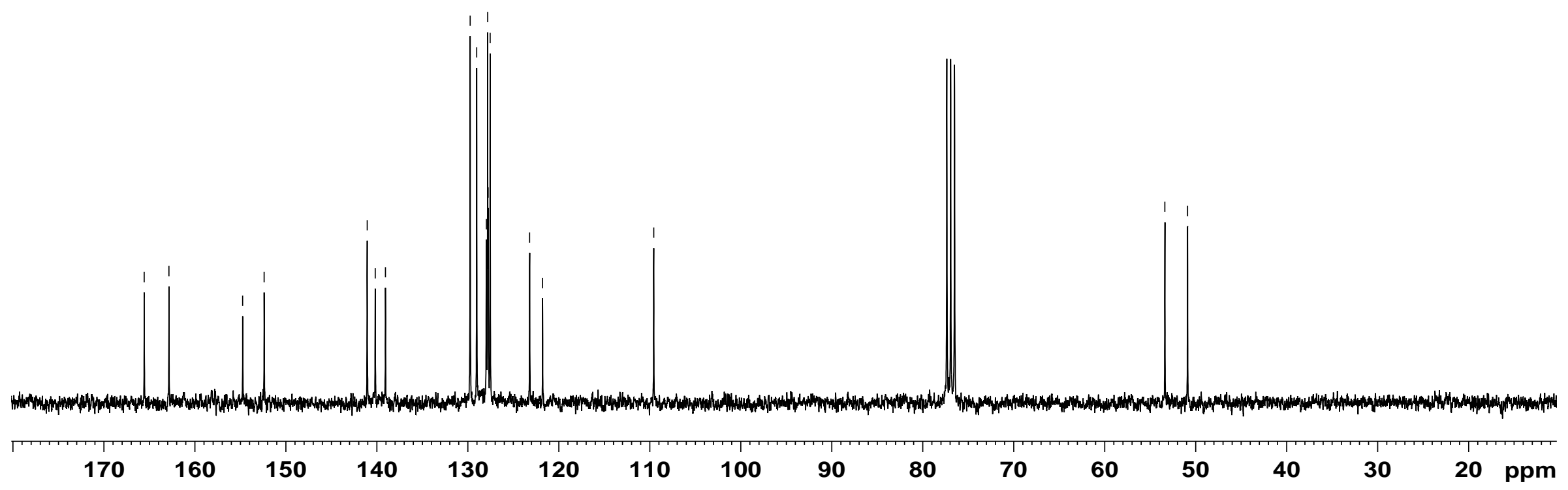




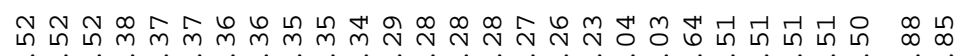

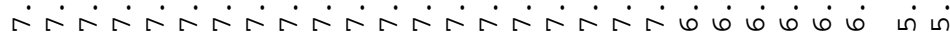

r
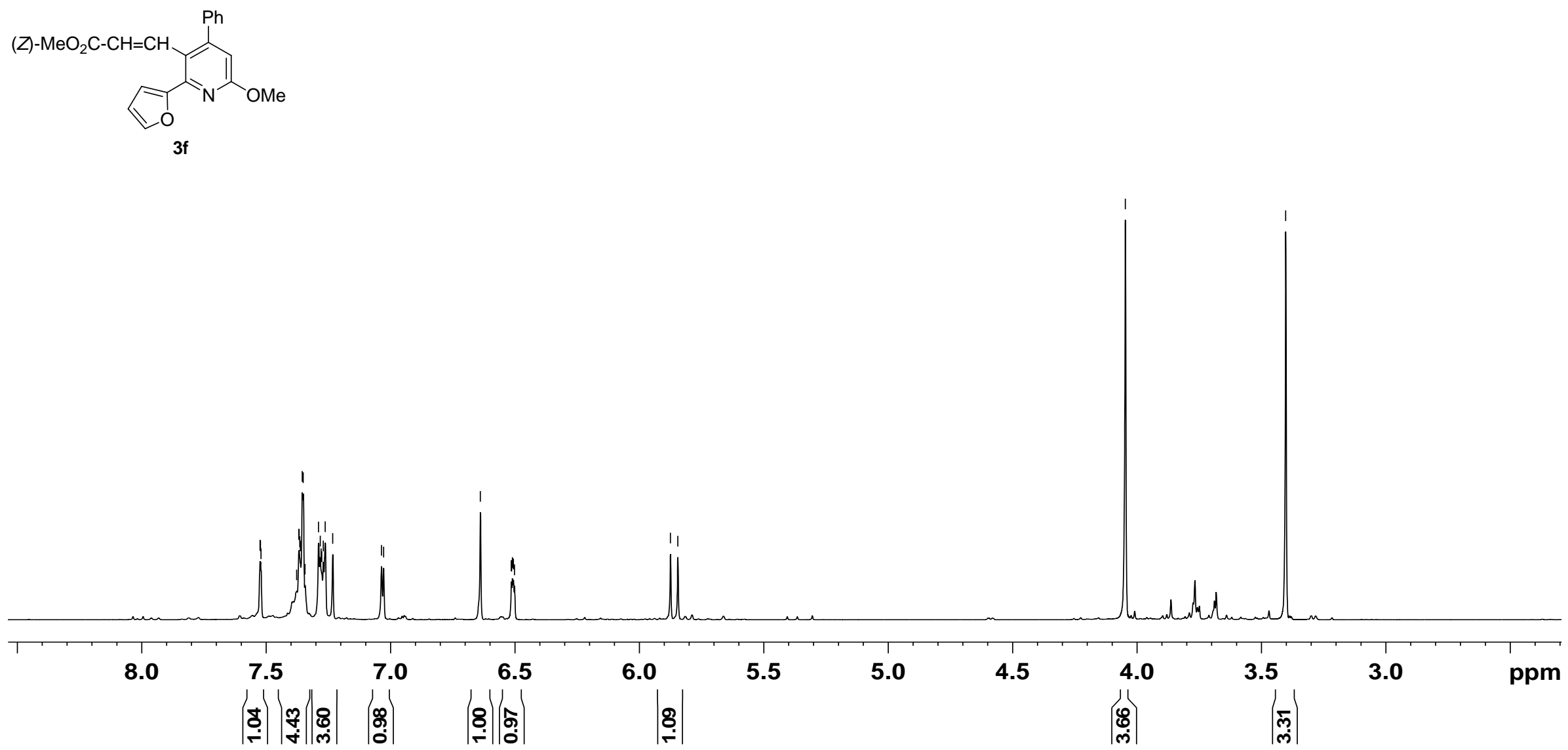


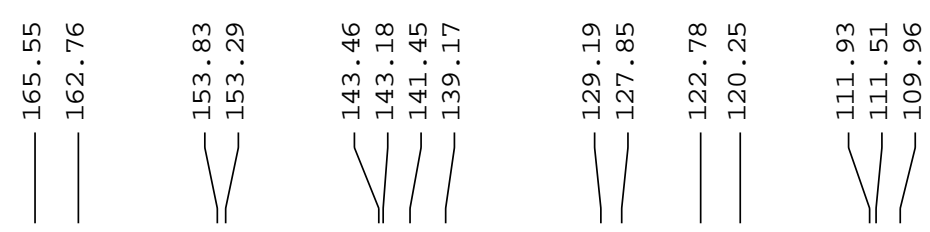

ชิ ธี

กิ

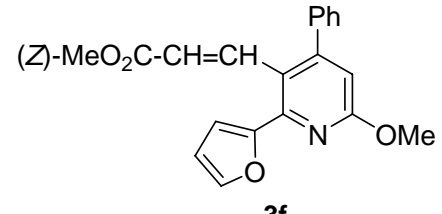

$3 f$

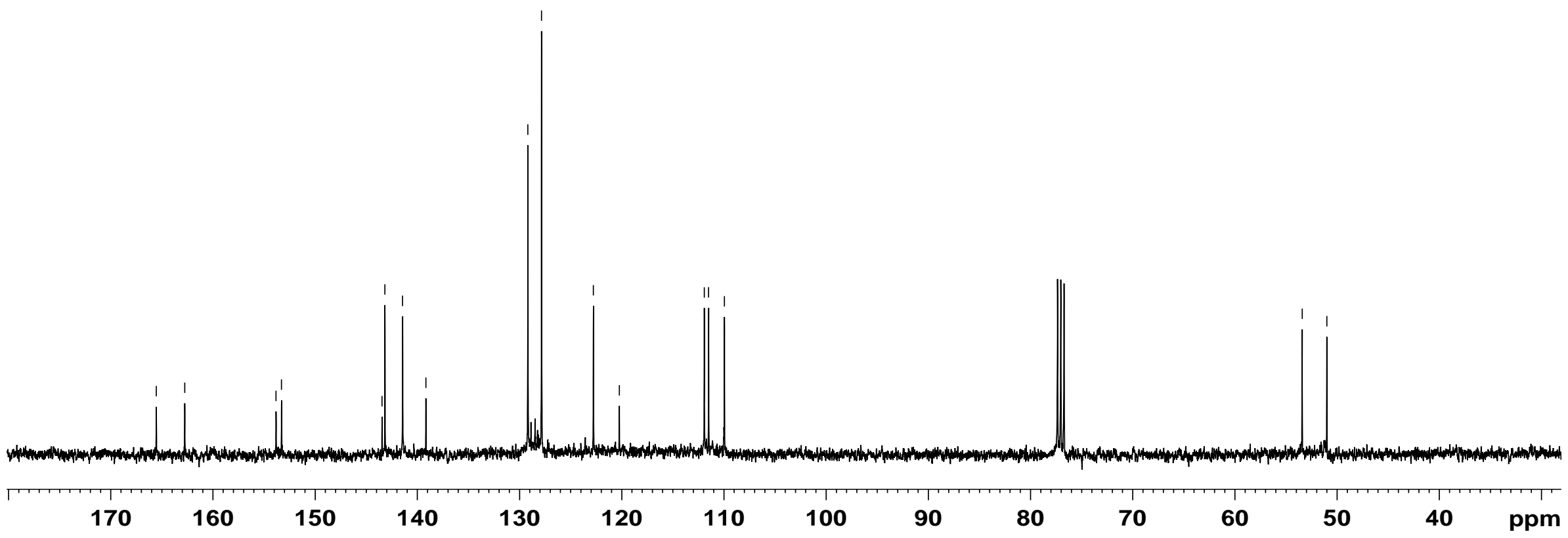




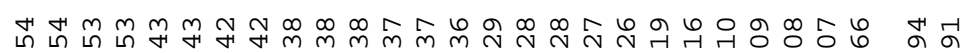

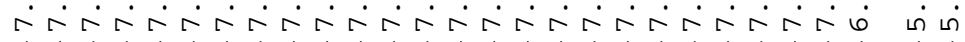

L

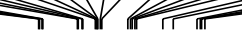

(Z) $-\mathrm{MeO}_{2} \mathrm{C}-\mathrm{CH}=\mathrm{CH}_{\mathrm{OMe}}$

$3 g$
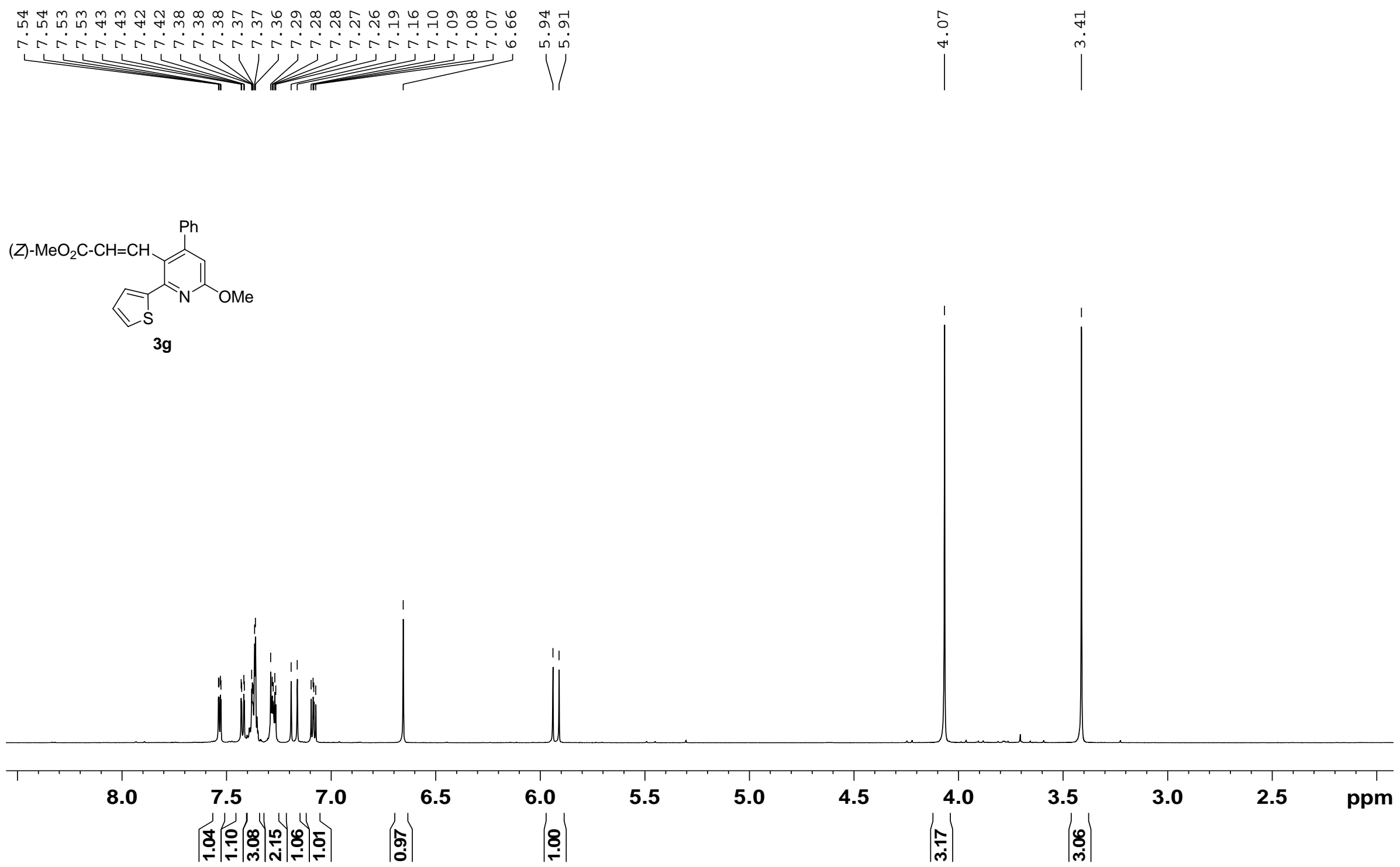

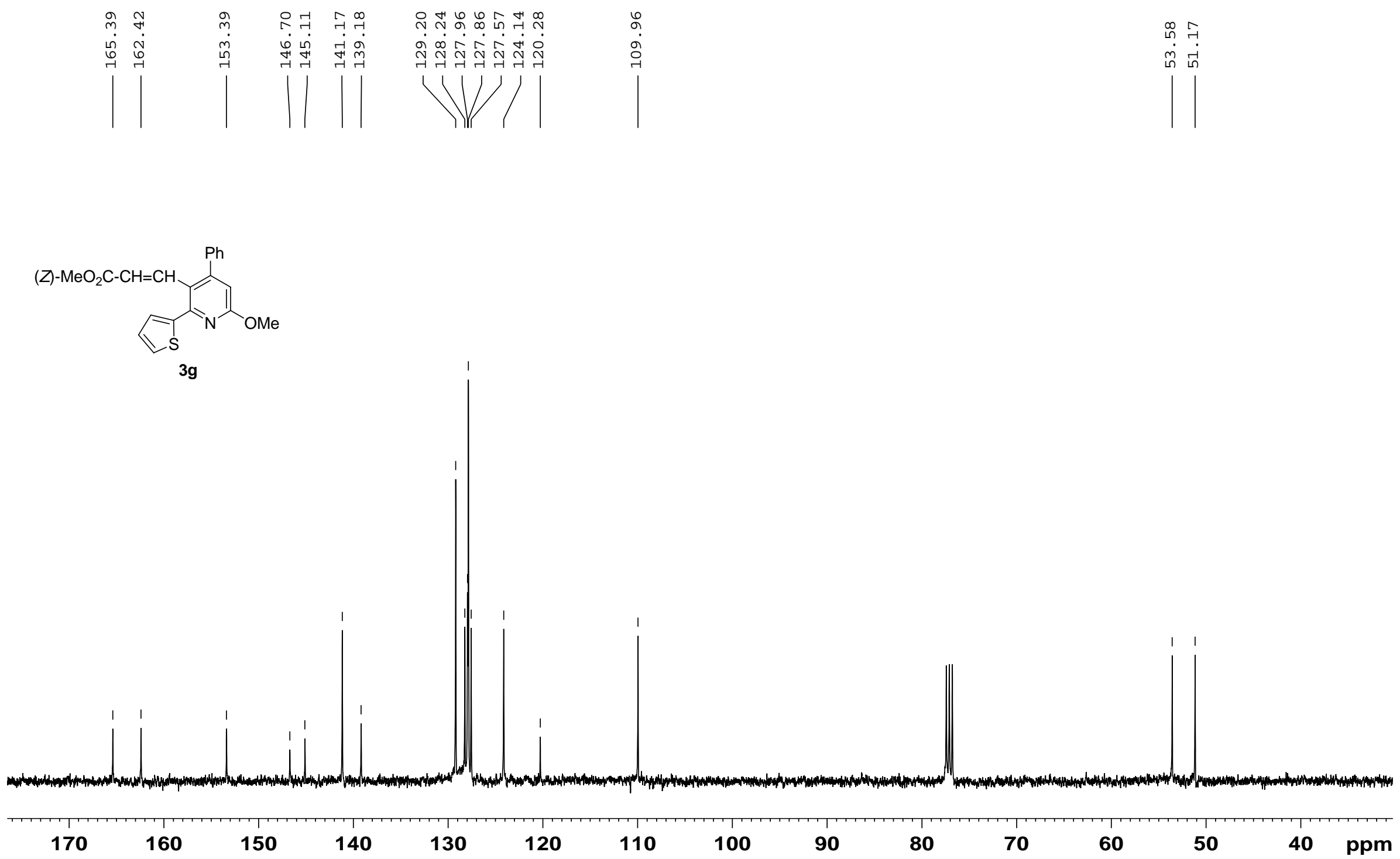


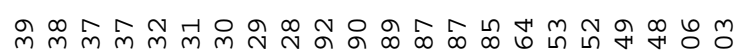

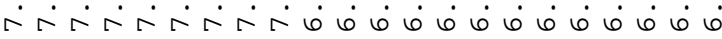

(l)
ㅇำ ㅇำ ๆ

101010

$1 /$

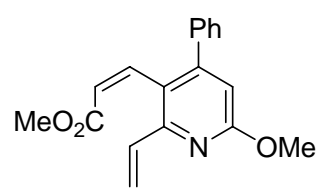

3h

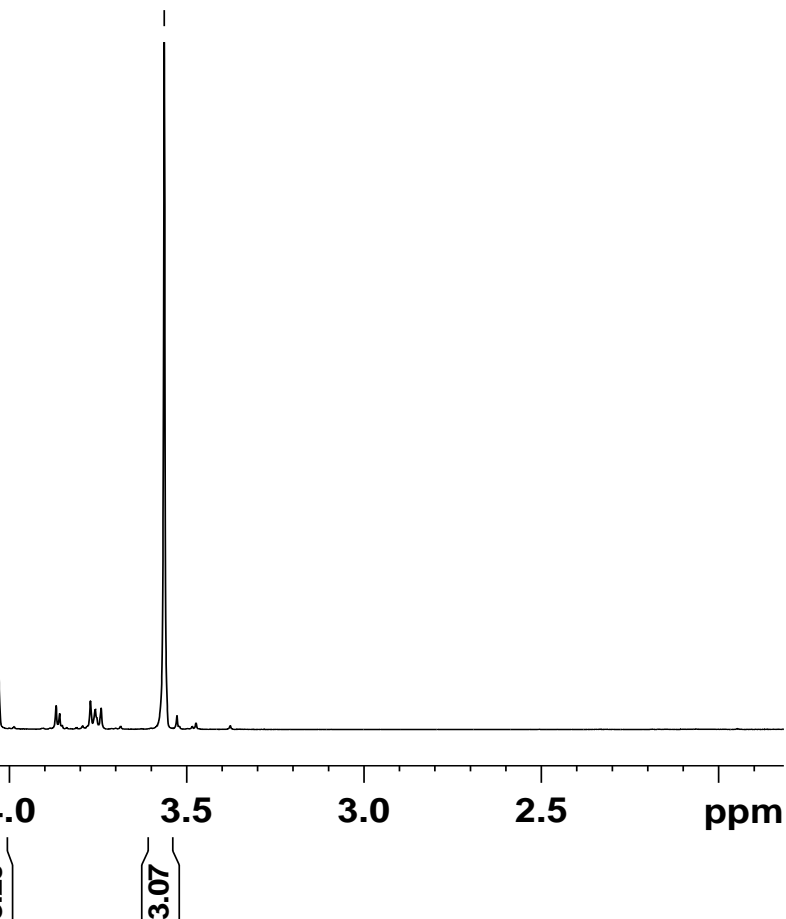




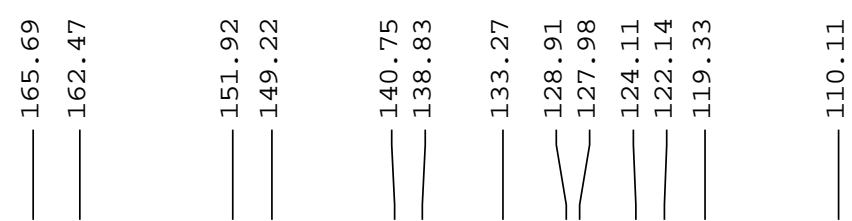

궁

लिं

1

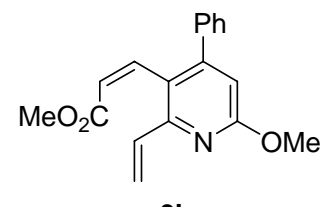

3h

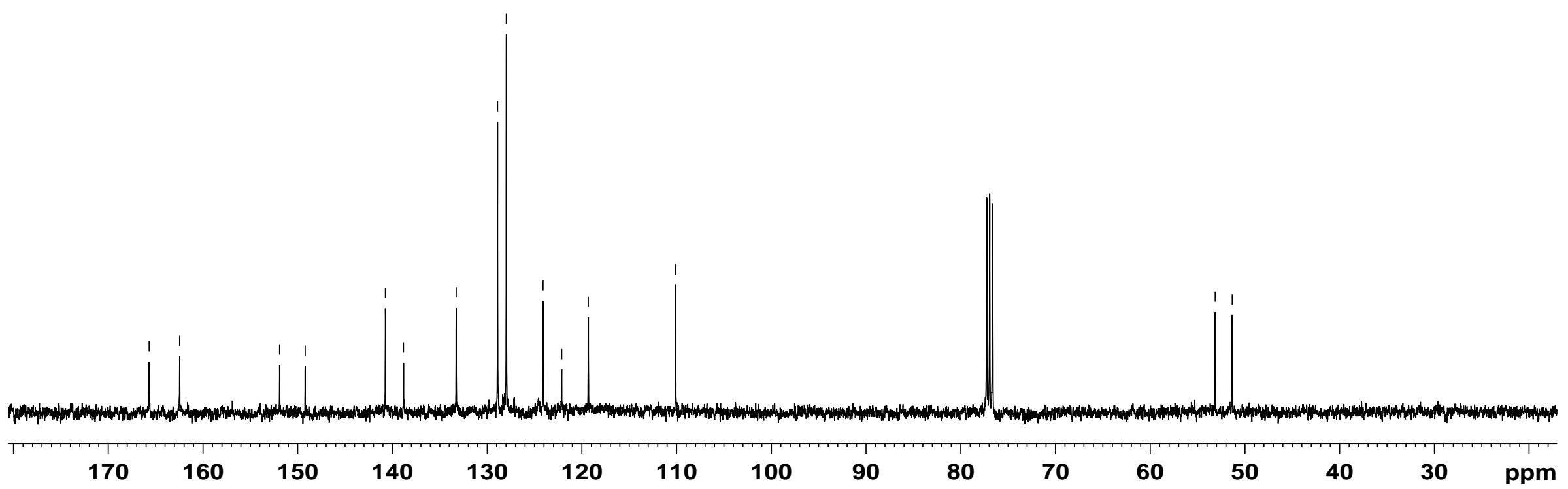



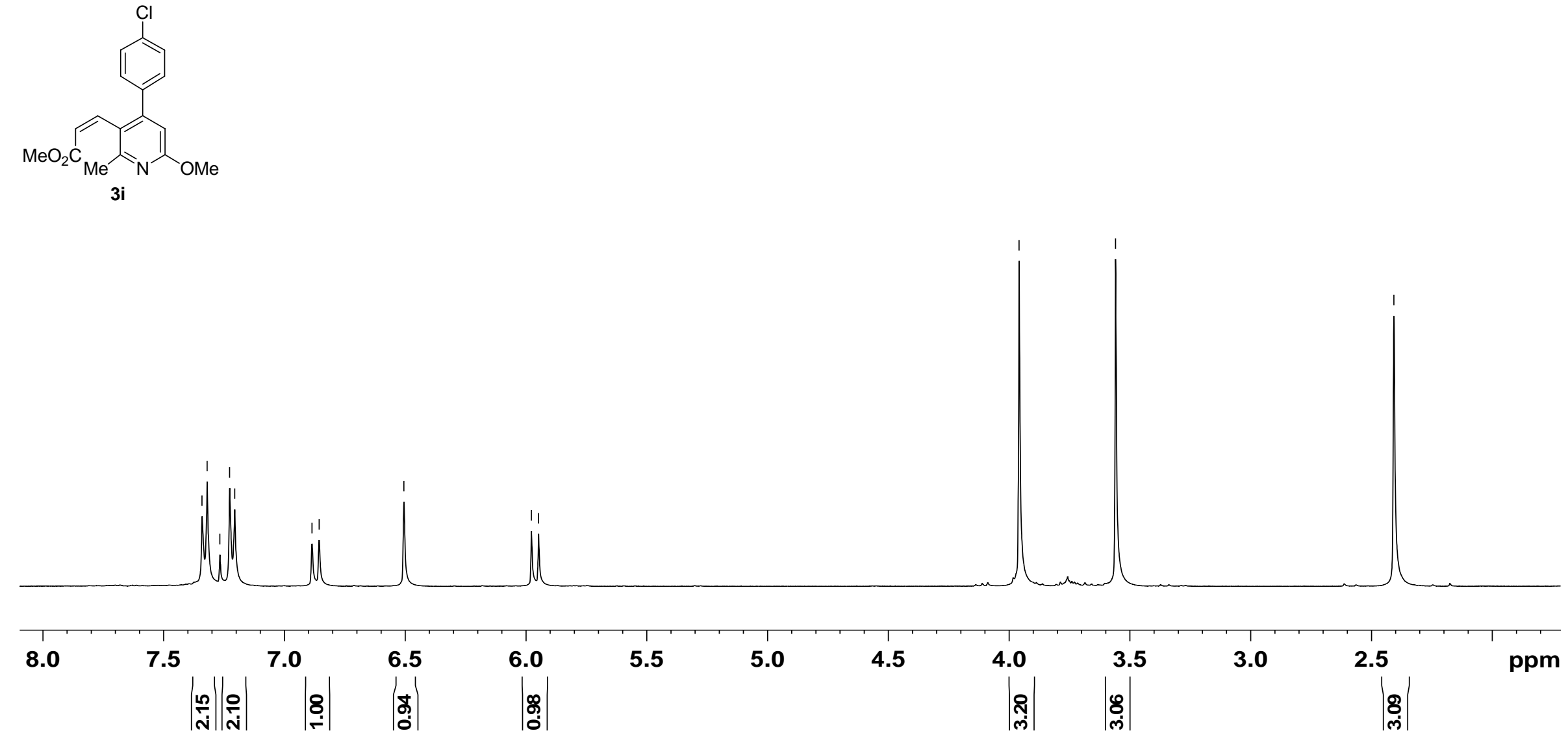

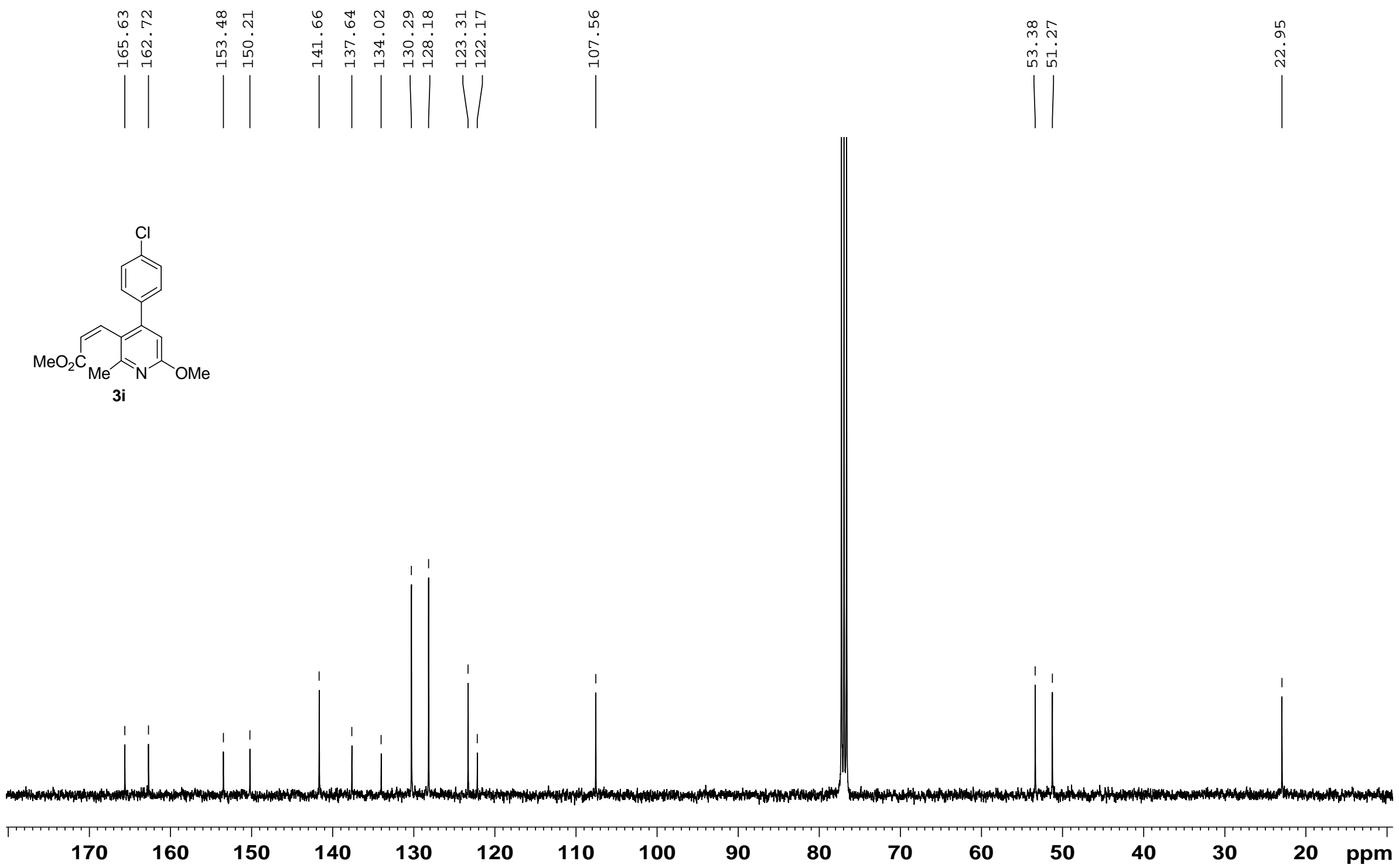


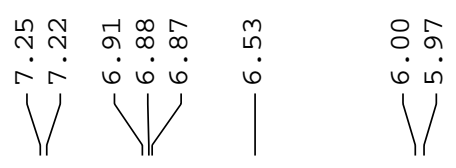

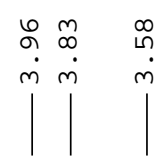

ㅁำ

N
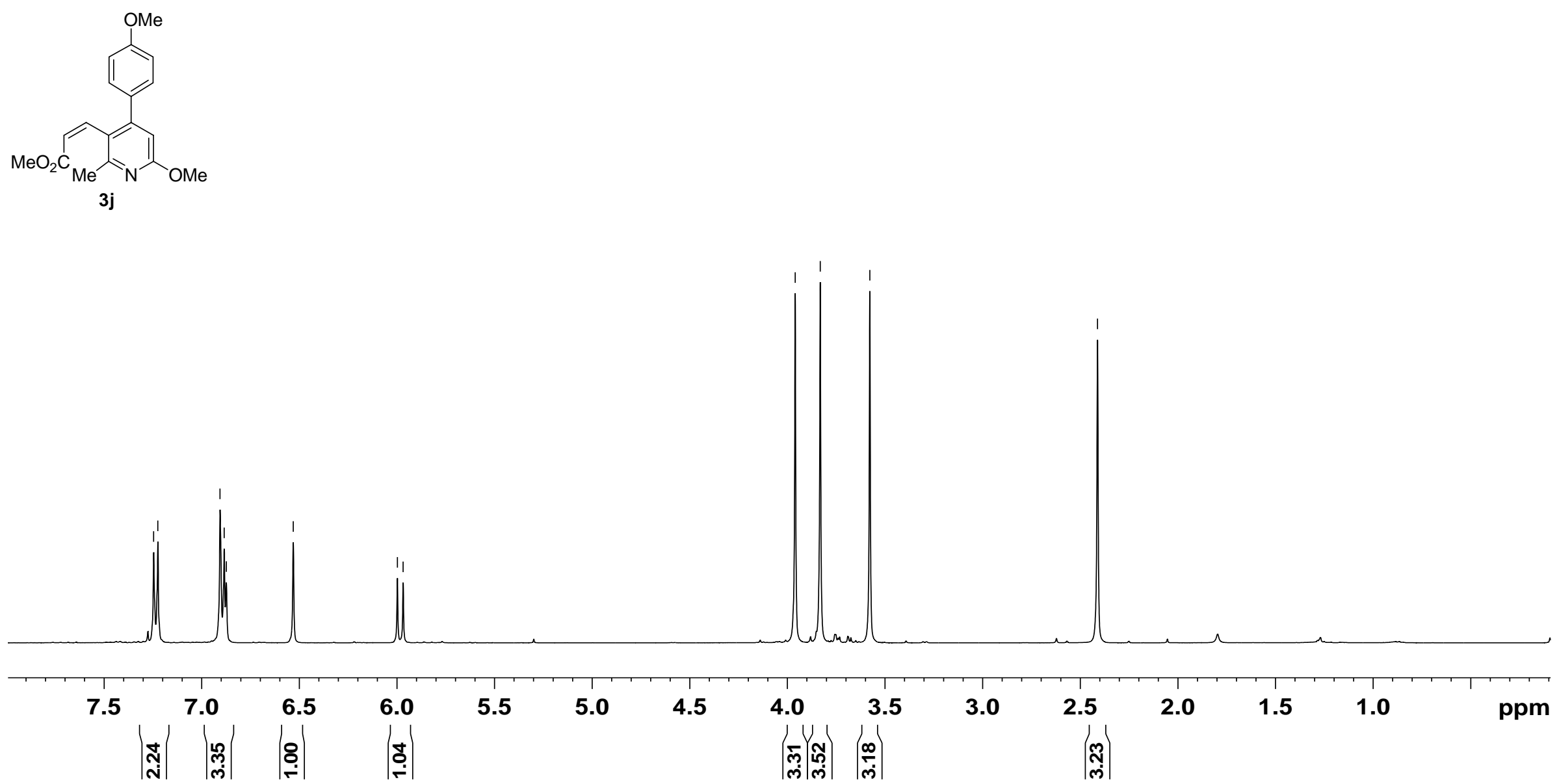

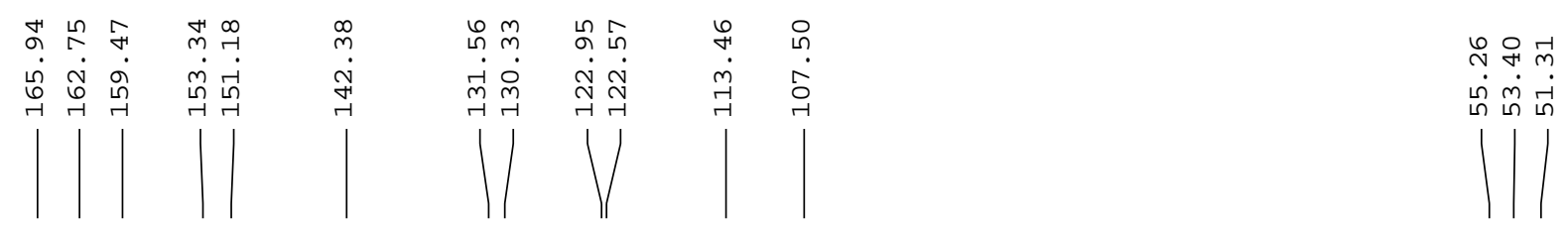

กำ

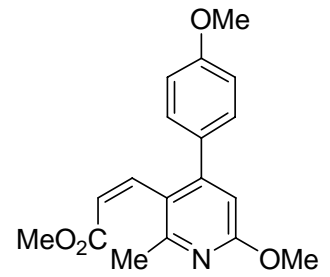

3j

(1) 


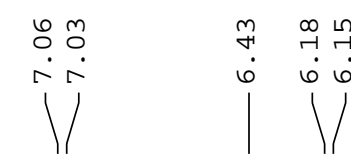

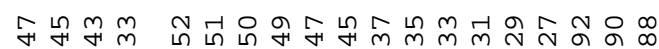

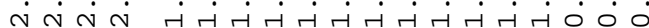
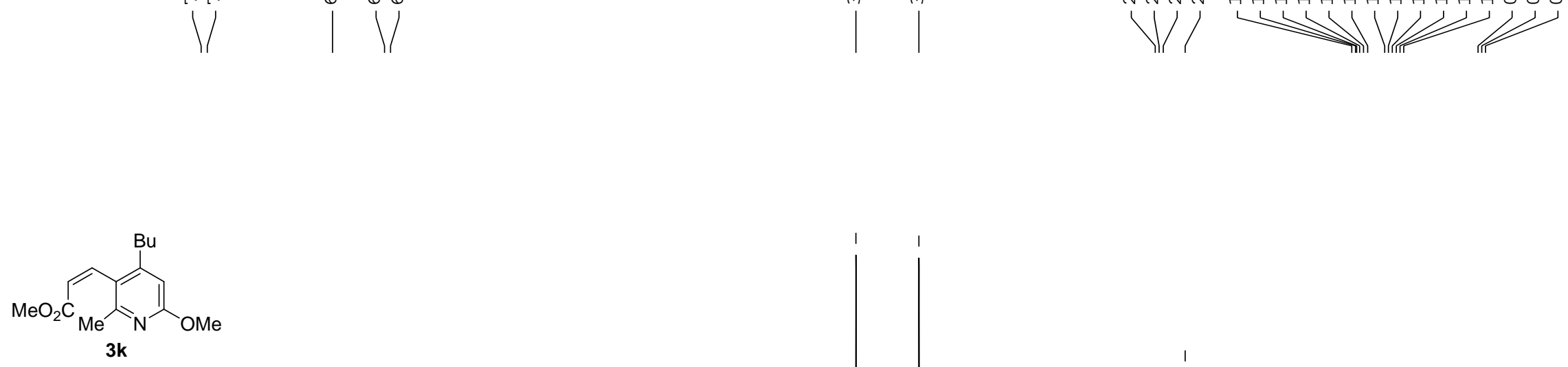

3k
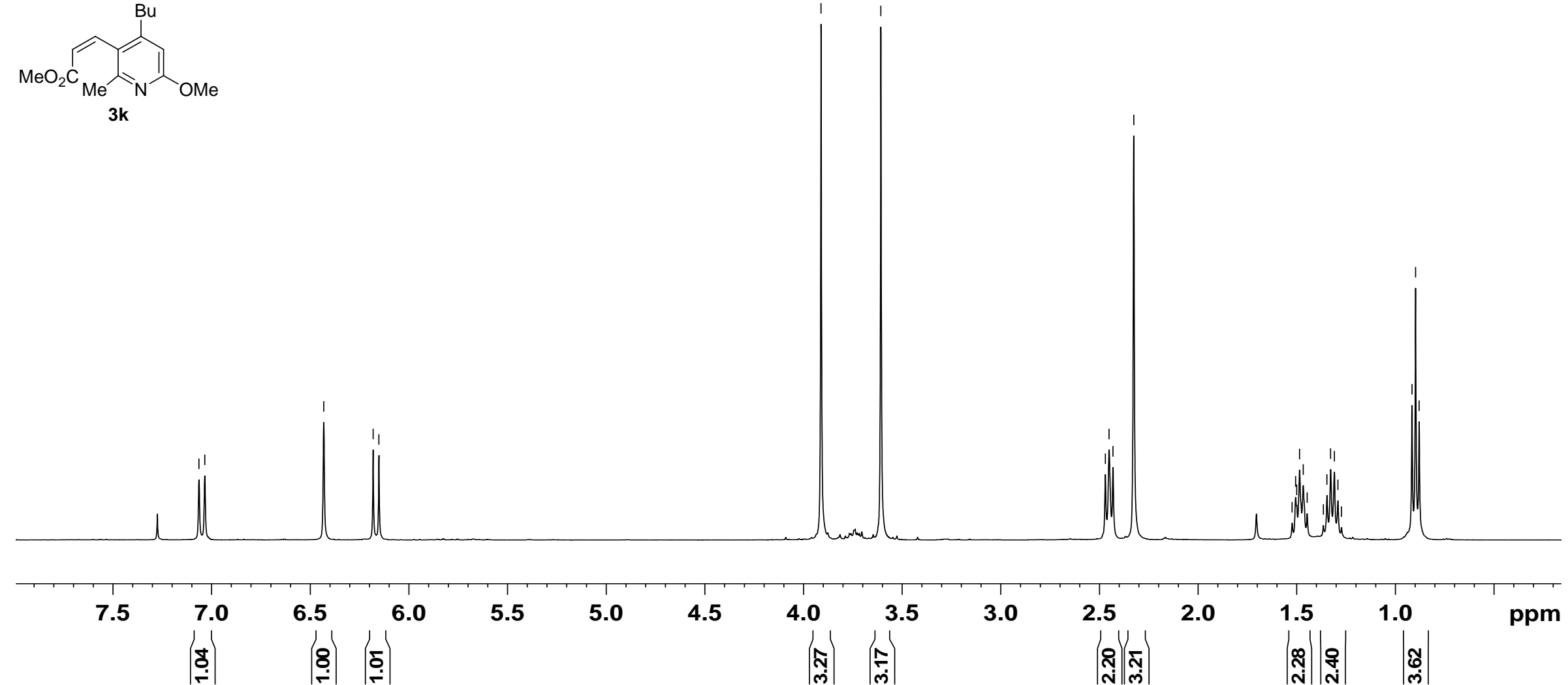

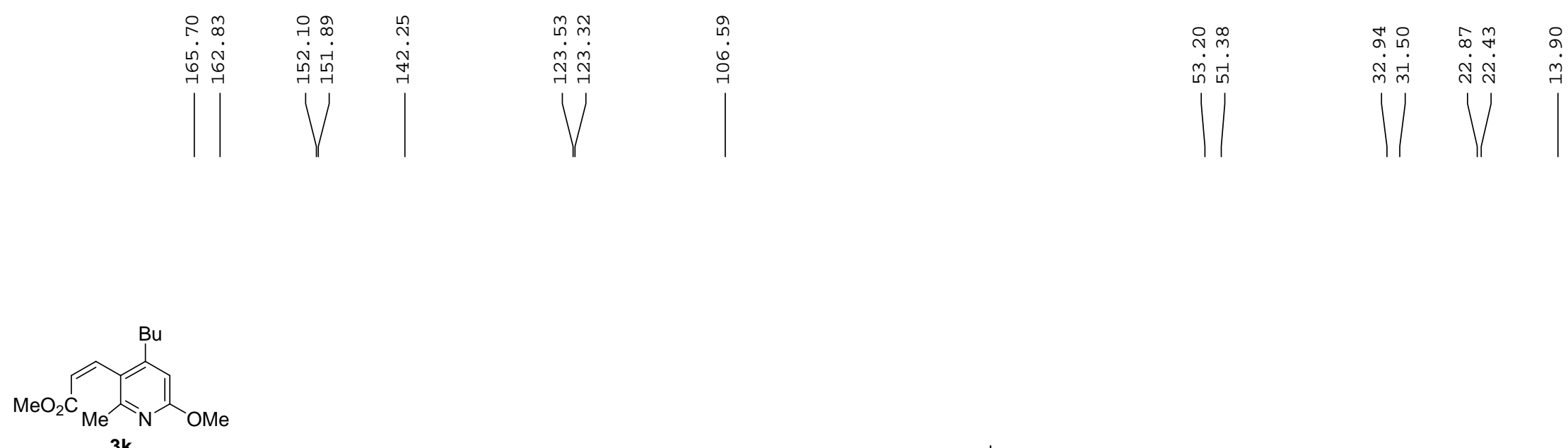

3k

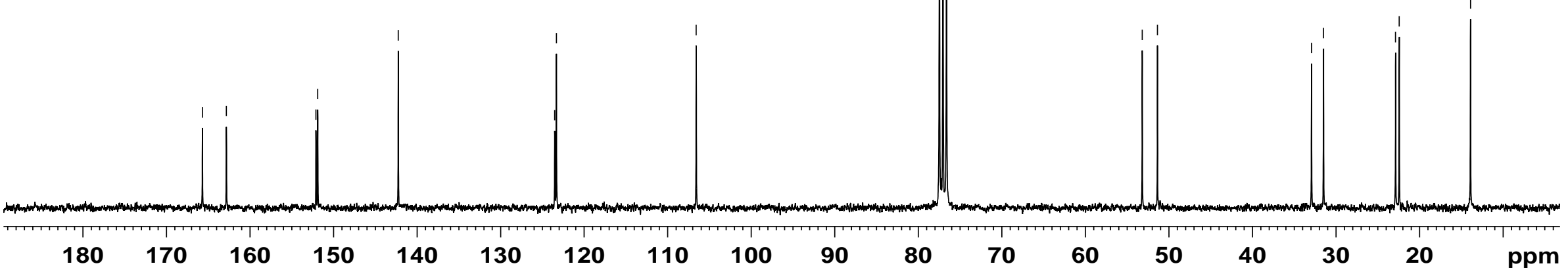




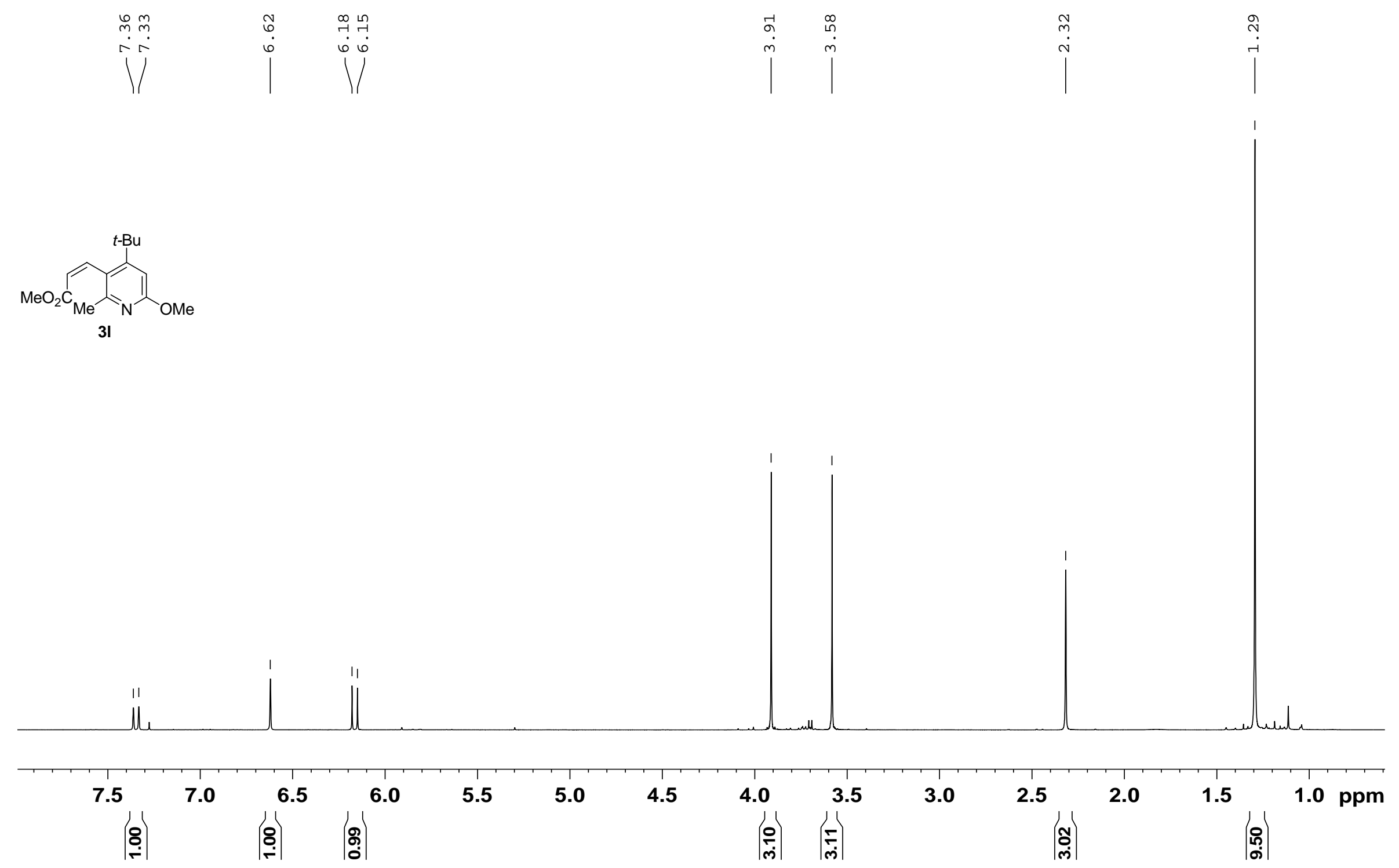



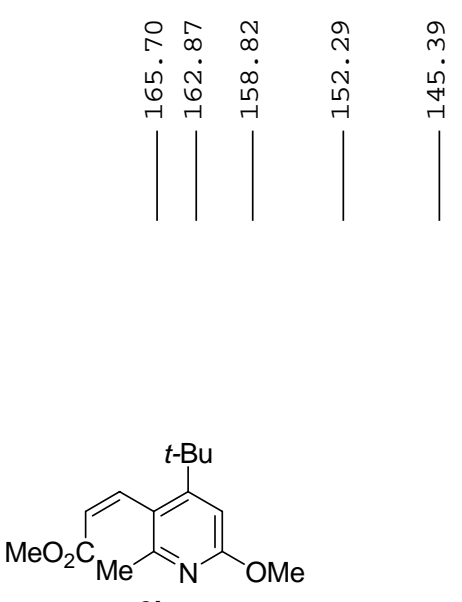

3।

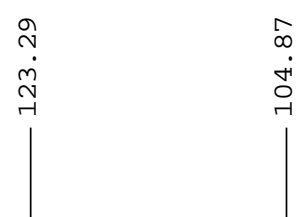

구

苚

1

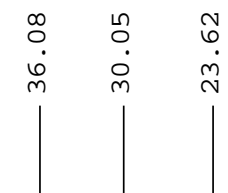

$\begin{array}{lll}\infty & 0 & 0\end{array}$

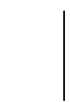

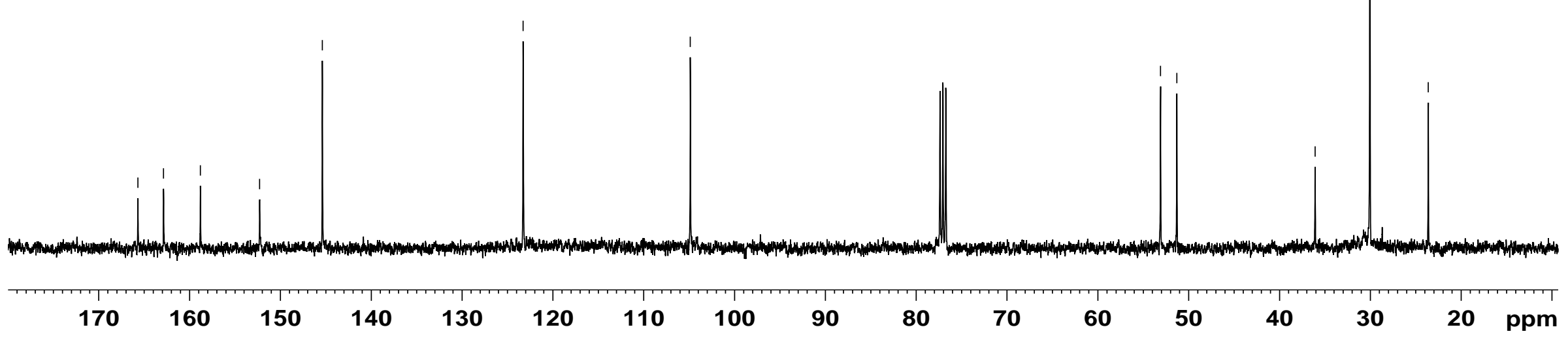



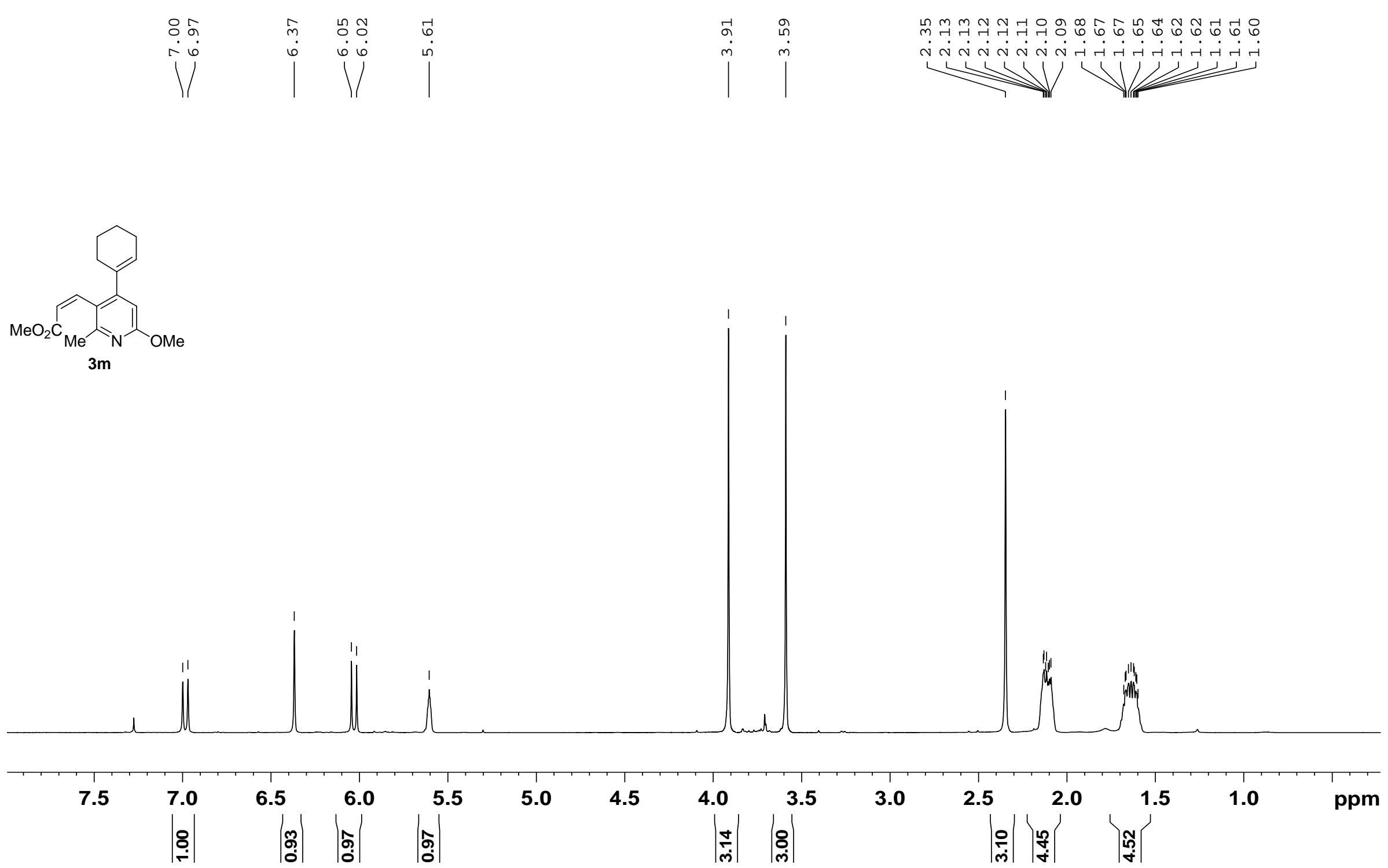

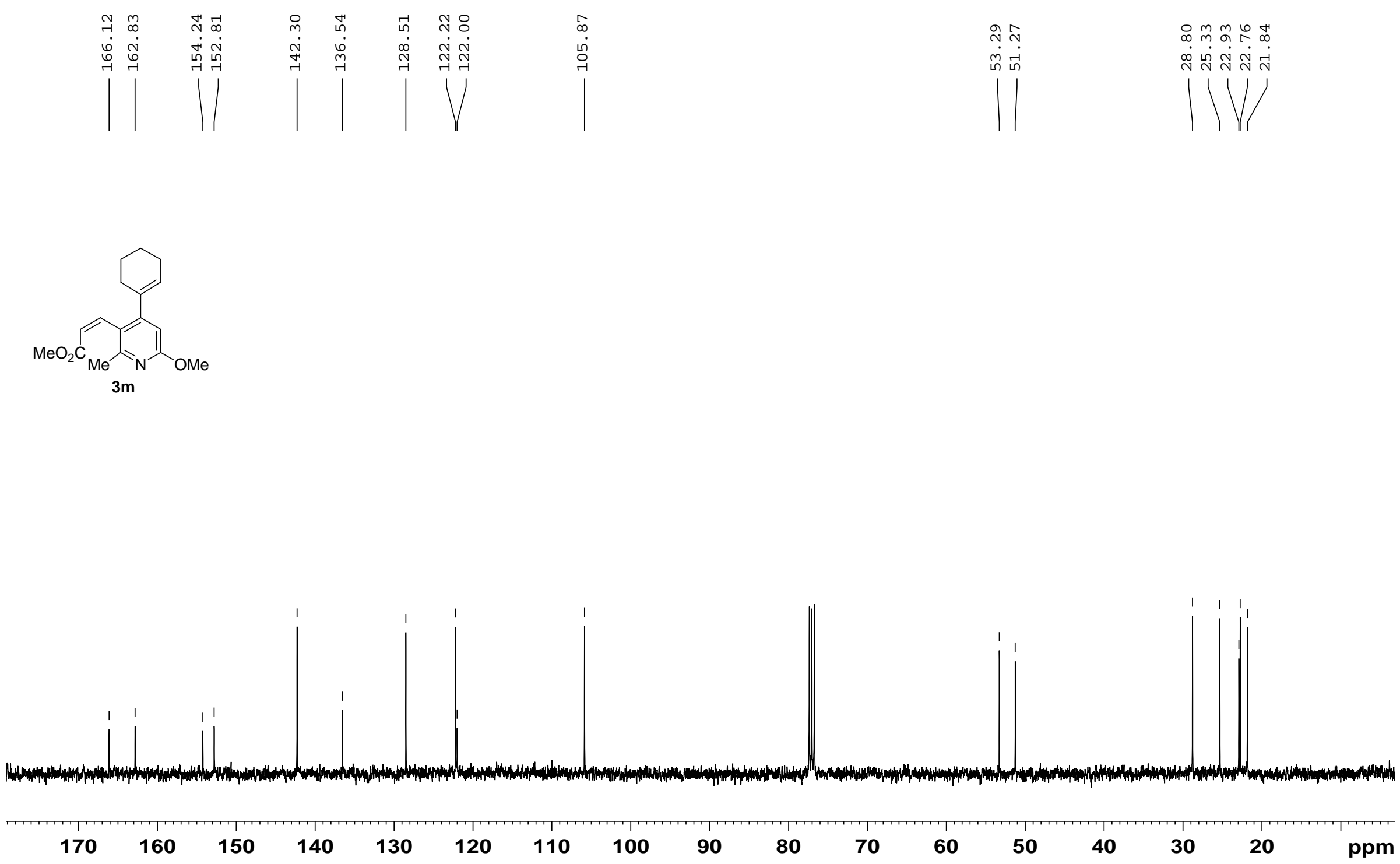

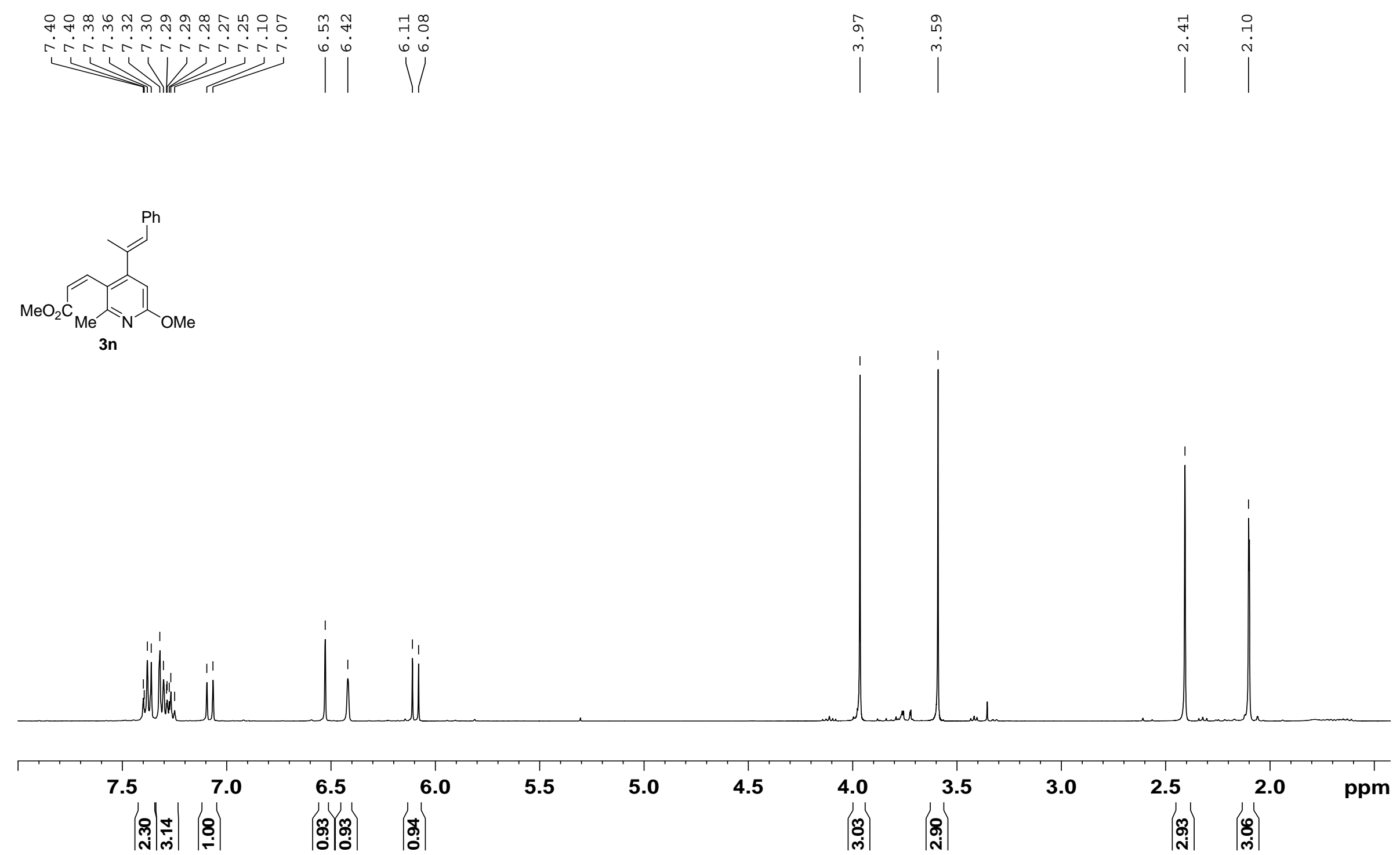


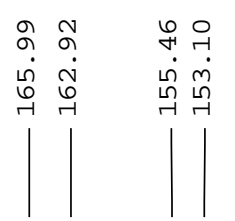

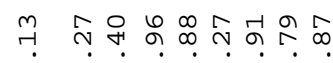

ปั

กิ

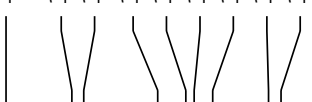

कृष

लि

ค. ๕

$\mid$

กิ สำ

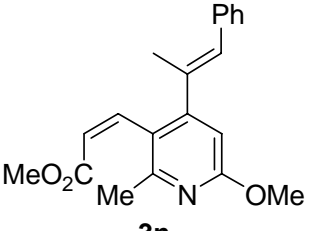

$3 n$

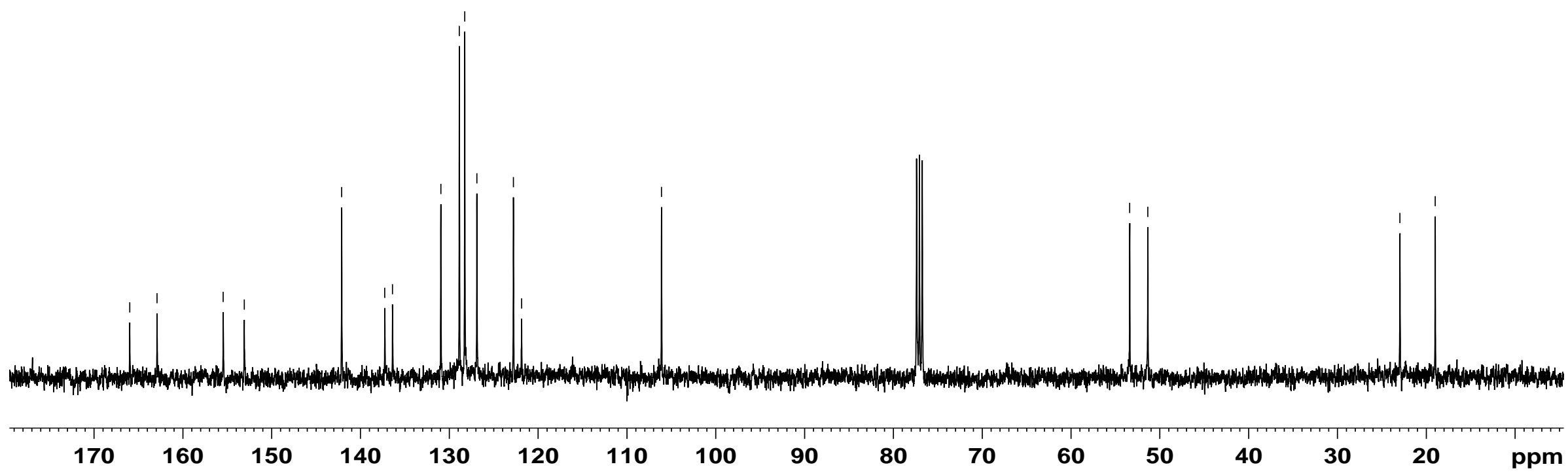



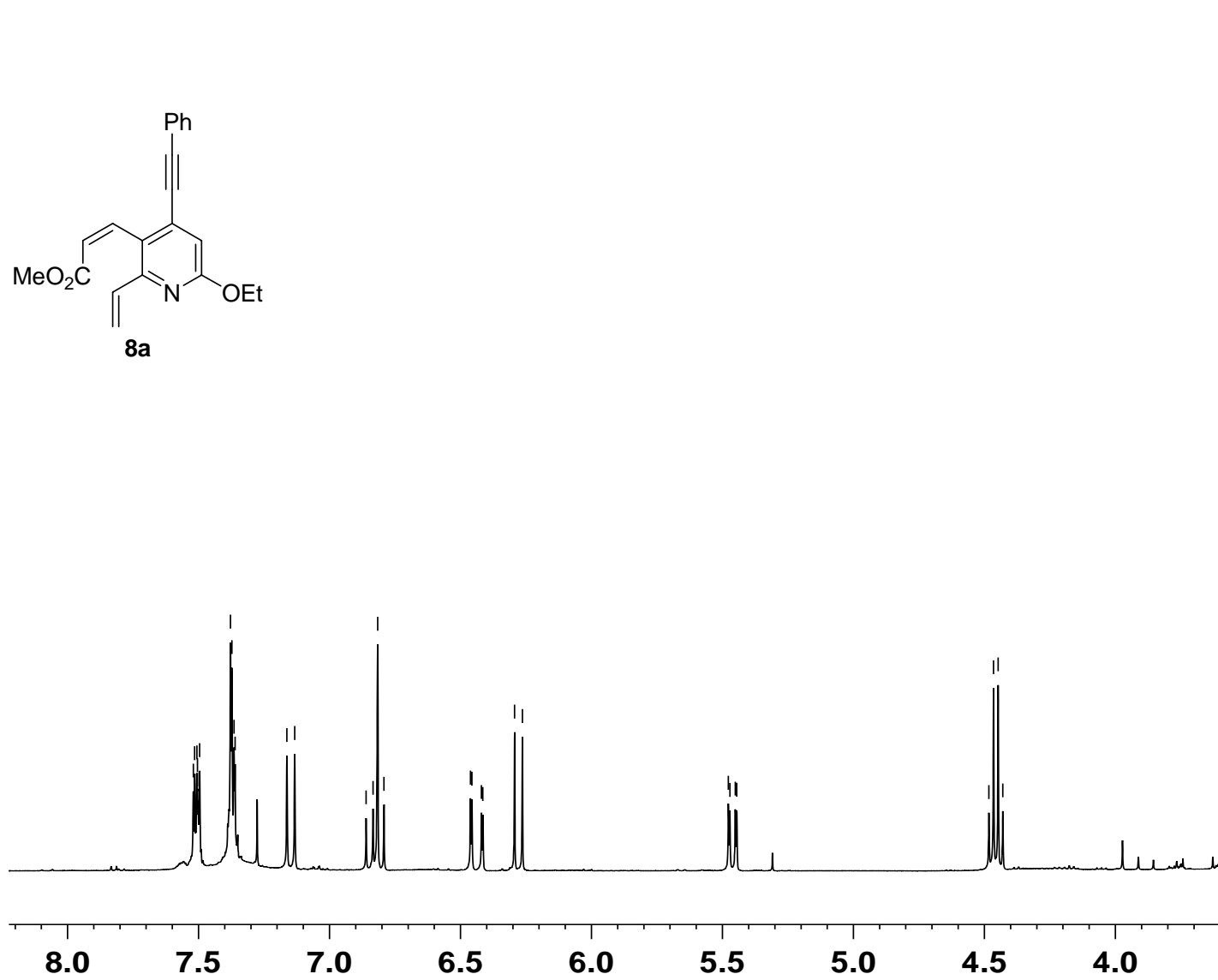
6.5

6.0

5.5

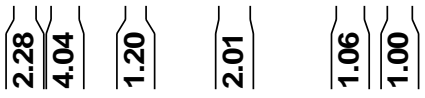

$\left|\begin{array}{l}\text { o } \\ \text { i }\end{array}\right|$

4.5

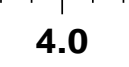

3.5

$\left|\begin{array}{l}n \\ m\end{array}\right|$

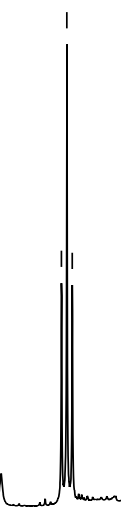




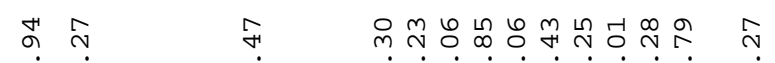

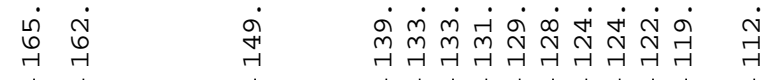

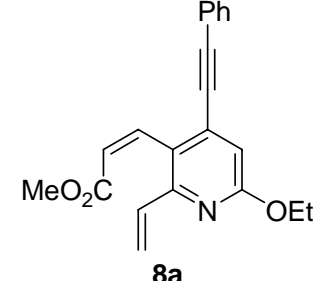

$8 a$

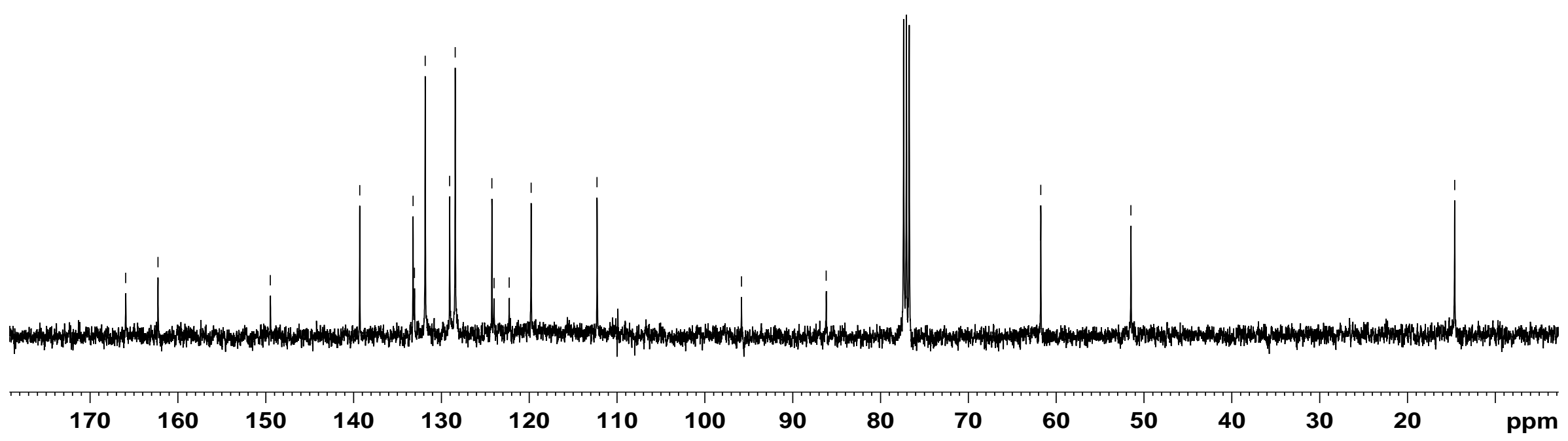




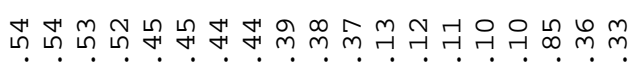

risinisinisitikiogo

$\longrightarrow$

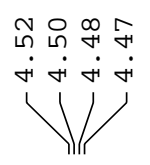

ก)

舟 过昌

$\sqrt{4-1}$

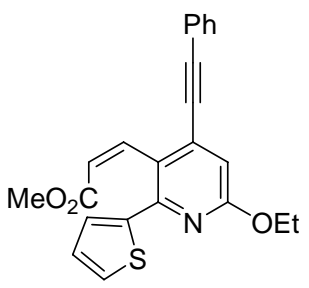

$8 b$

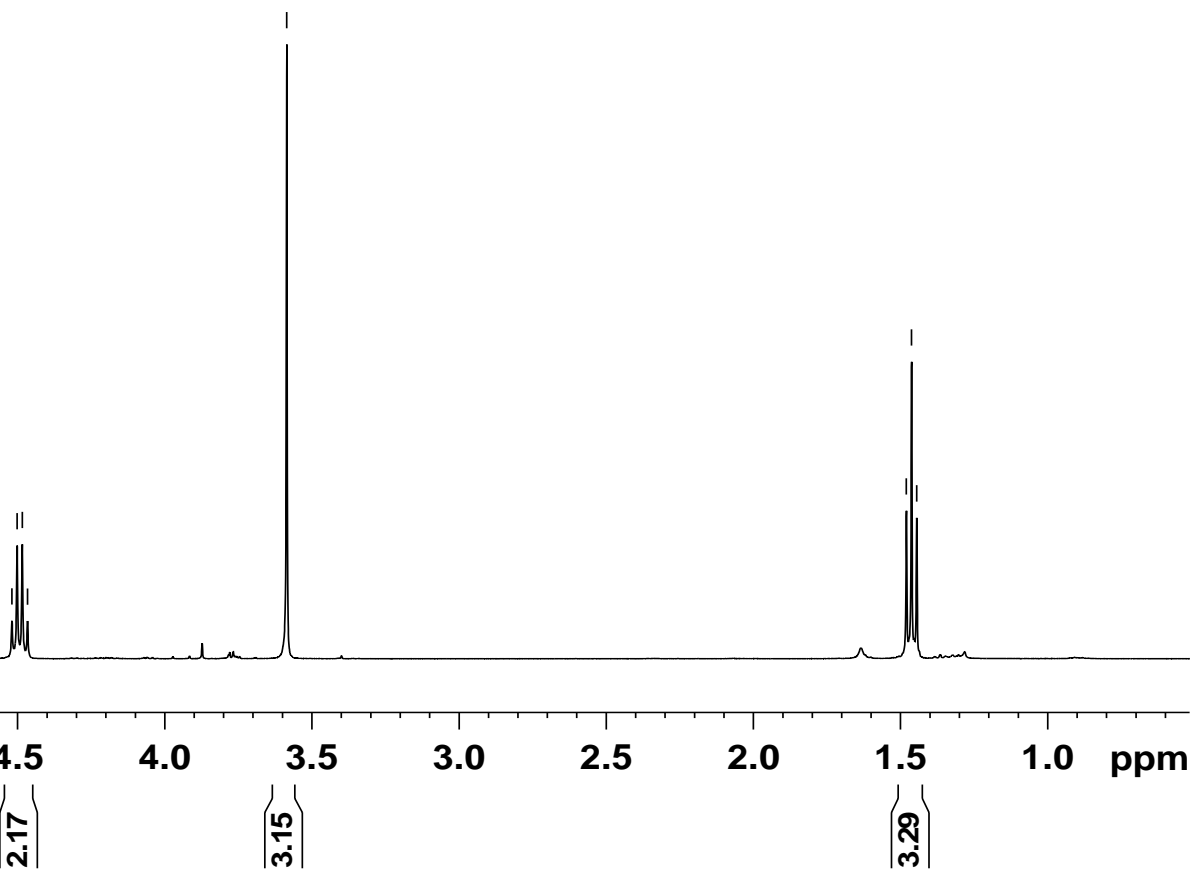



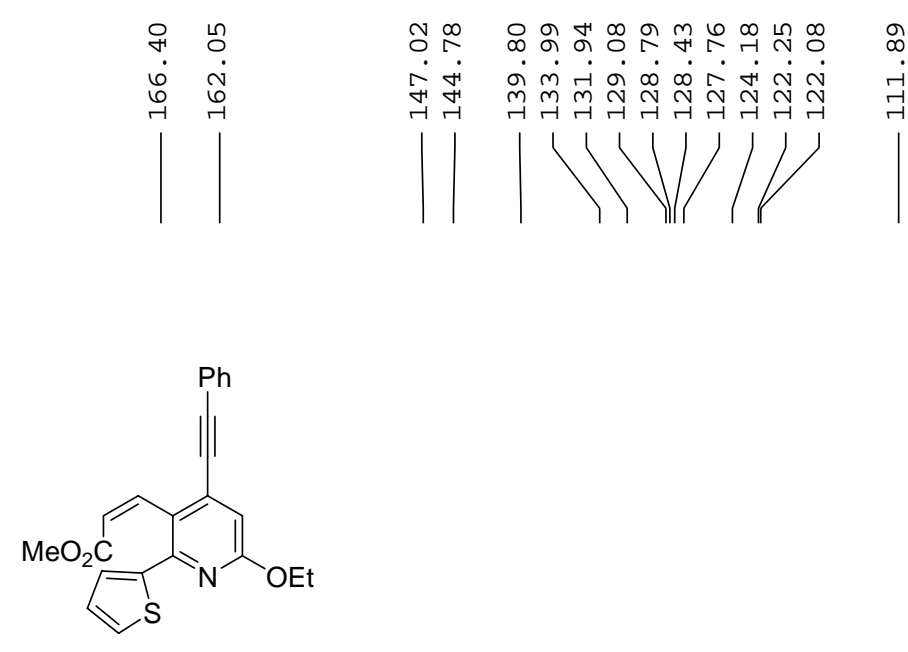

$8 b$

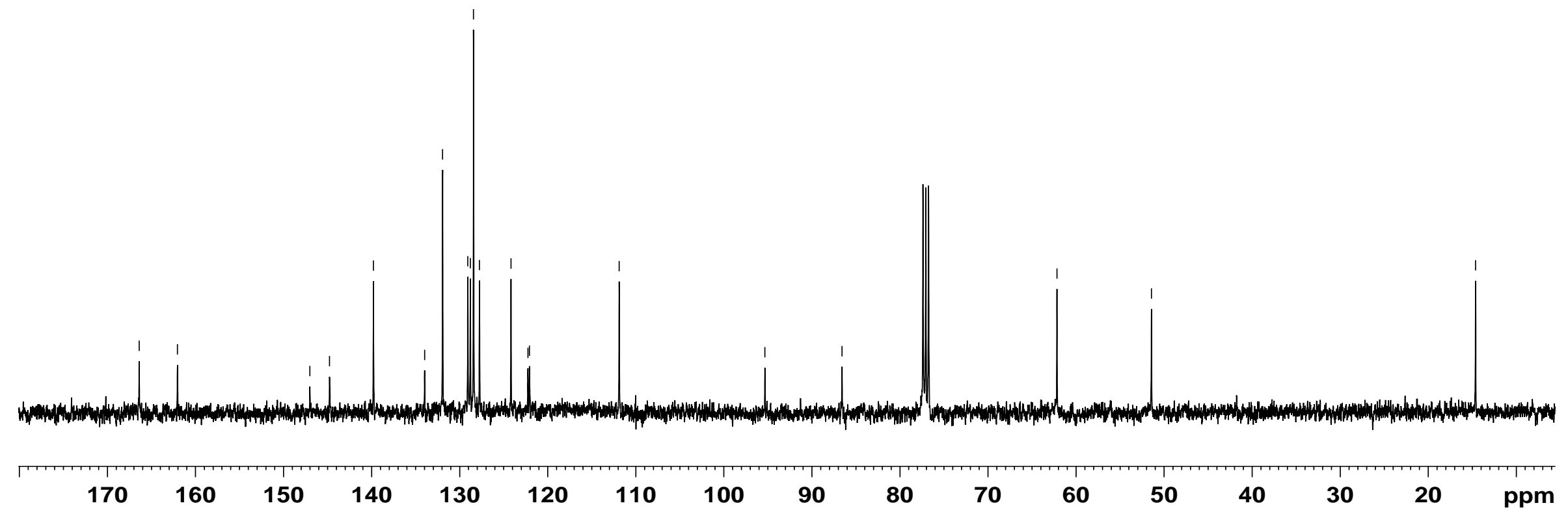




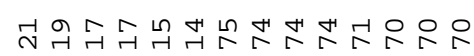

$\dot{0} \dot{0} \dot{0} \dot{0} \dot{0}$ ம

$+1$ $\operatorname{lng} 2$

लंखिं लं

I/

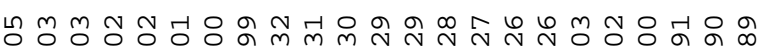

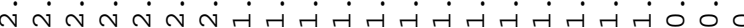

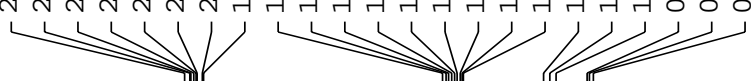

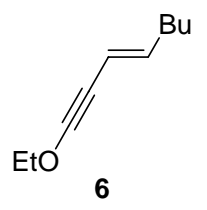

$\mathrm{Bu}$

6

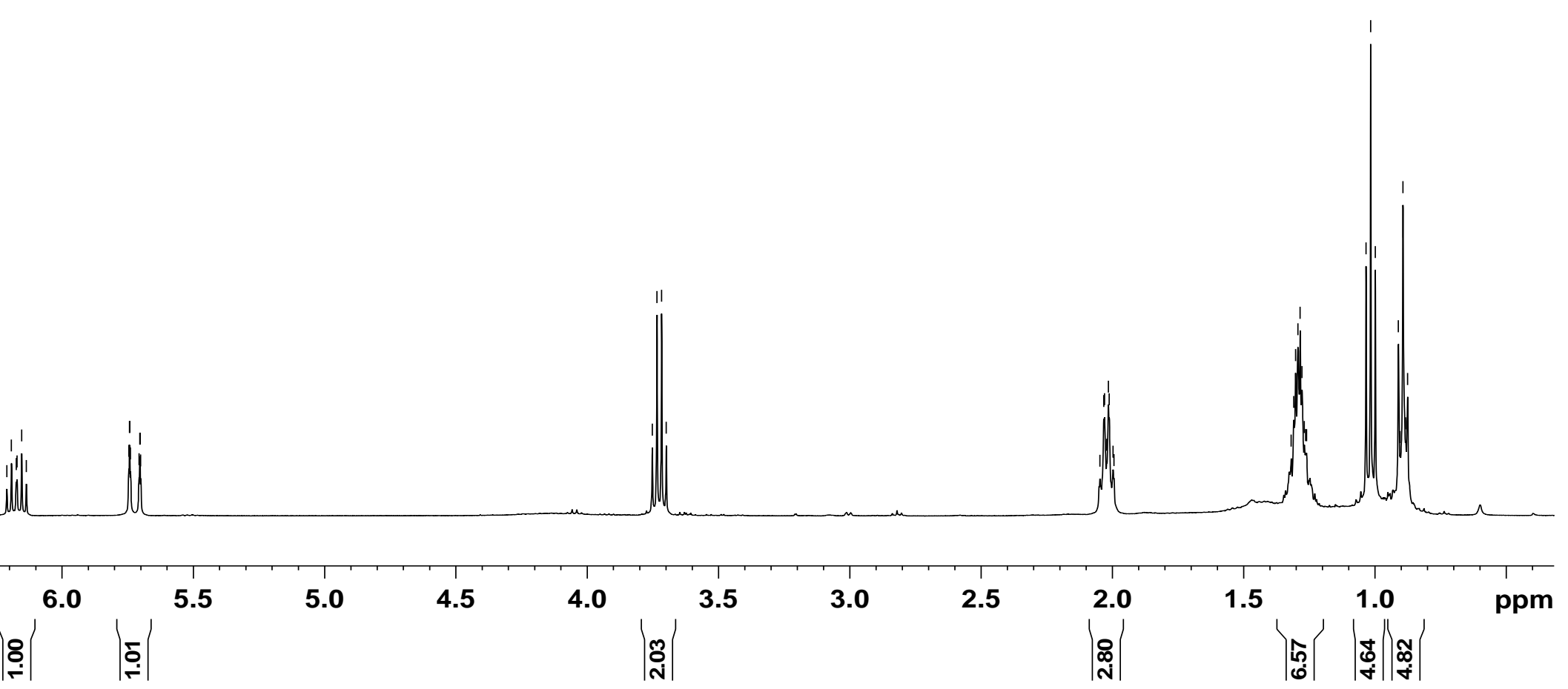




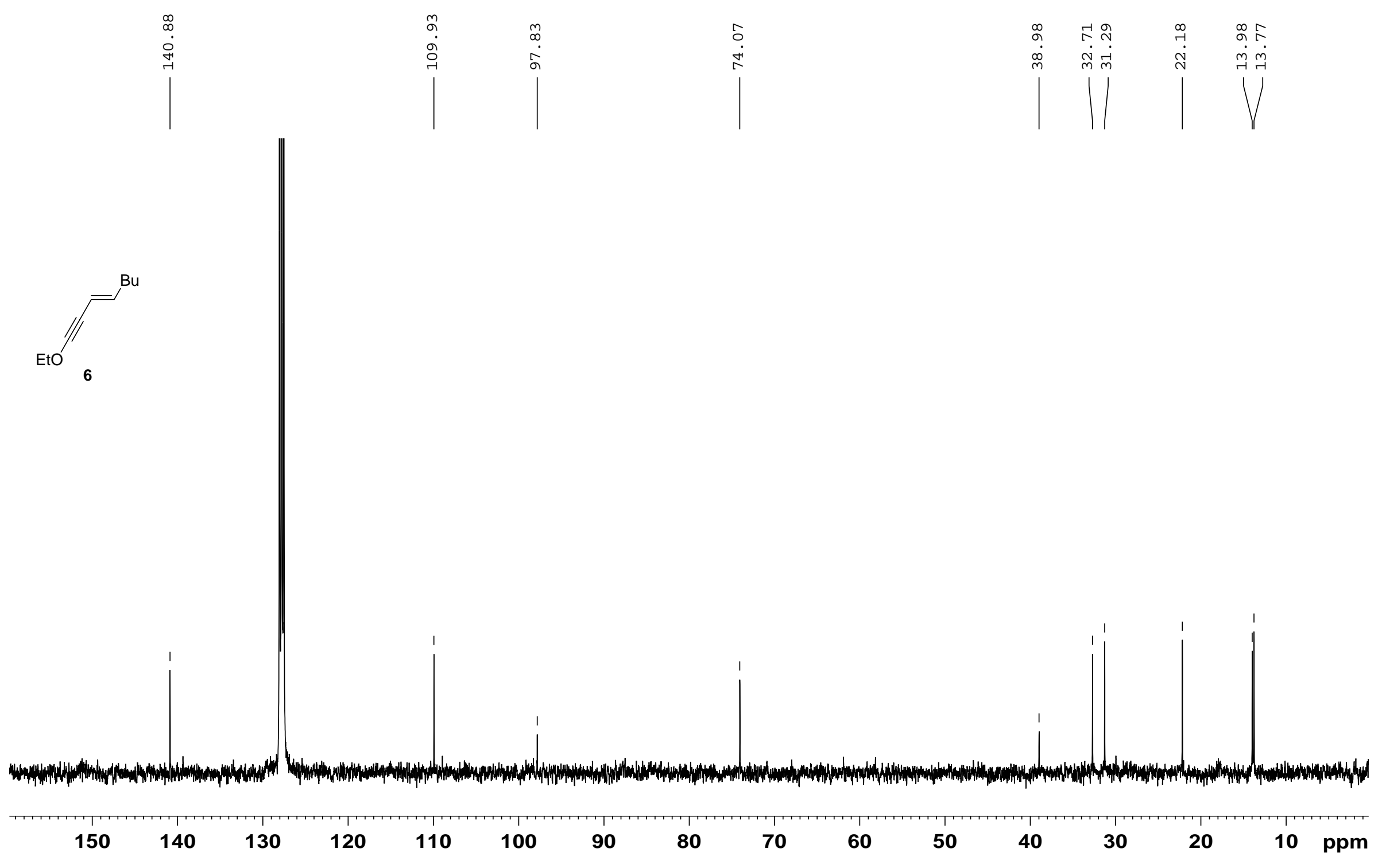

